\title{
Candesartan prevents arteriopathy progression in cerebral autosomal recessive arteriopathy with subcortical infarcts and leukoencephalopathy model
}

\begin{abstract}
Taisuke Kato, ${ }^{1}$ Ri-ichiroh Manabe, ${ }^{2}$ Hironaka Igarashi, ${ }^{3}$ Fuyuki Kametani, ${ }^{4}$ Sachiko Hirokawa, ${ }^{5}$ Yumi Sekine, ${ }^{5}$ Natsumi Fujita, Satoshi Saito, ${ }^{6}$ Yusuke Kawashima, ${ }^{7}$ Yuya Hatano, ${ }^{5}$ Shoichiro Ando, ${ }^{5}$ Hiroaki Nozaki, ${ }^{8}$ Akihiro Sugai, ${ }^{5}$ Masahiro Uemura, ${ }^{5}$ Masaki Fukunaga, ${ }^{9}$ Toshiya Sato, ${ }^{10}$ Akihide Koyama, ${ }^{11}$ Rie Saito, ${ }^{12}$ Atsushi Sugie, ${ }^{13}$ Yasuko Toyoshima, ${ }^{12}$ Hirotoshi Kawata, ${ }^{14}$ Shigeo Murayama, ${ }^{15,16}$ Masaki Matsumoto, ${ }^{17}$ Akiyoshi Kakita, ${ }^{12}$ Masato Hasegawa, ${ }^{4}$ Masafumi Ihara, ${ }^{6}$ Masato Kanazawa, ${ }^{5}$ Masatoyo Nishizawa, ${ }^{18}$ Shoji Tsuji, ${ }^{19}$ and Osamu Onodera ${ }^{5}$

'Department of System Pathology for Neurological Disorders, Brain Science Branch, Brain Research Institute, Niigata University, Niigata, Japan. ²aboratory for Comprehensive Genomic Analysis, Center for Integrative Medical Sciences, RIKEN, Kanagawa, Japan. ${ }^{3}$ Center for Integrated Human Brain Science, Brain Research Institute, Niigata University, Niigata, Japan. ${ }^{4}$ Department of Brain and Neuroscience, Tokyo Metropolitan Institute of Medical Science, Tokyo, Japan. ${ }^{5}$ Department of Neurology, Clinical Neuroscience Branch, Brain Research Institute, Niigata University, Niigata, Japan. ${ }^{6}$ Department of Neurology, National Cerebral and Cardiovascular Center, Suita, Japan. ${ }^{7}$ Department of Applied Genomics, Kazusa DNA Research Institute, Chiba, Japan. ${ }^{8}$ Department of Medical Technology, Graduate School of Health Sciences, Niigata University, Niigata, Japan. ${ }^{9}$ Division of Cerebral Integration, Department of System Neuroscience, National Institute for Physiological Sciences, Aichi, Japan. ${ }^{10}$ Department of Laboratory Animal Science, Kitasato University School of Medicine, Kanagawa, Japan. "'Department of Legal Medicine, Niigata University Graduate School of Medical and Dental Sciences, Niigata, Japan. ${ }^{12}$ Department of Pathology, Clinical Neuroscience Branch and ${ }^{13}$ Department of Neuroscience of Disease, Brain Research Institute, Niigata University, Niigata, Japan. ${ }^{14}$ Department of Pathology, Jichi Medical University, Tochigi, Japan. ${ }^{15}$ Brain Bank for Aging Research, Tokyo Metropolitan Geriatric Hospital and Institute of Gerontology, Tokyo, Japan. ${ }^{16}$ Brain Bank for Neurodevelopmental, Neurological and Psychiatric Disorders, United Graduate School of Child Development, University of Osaka, Osaka, Japan. ${ }^{17}$ Department of Omics and Systems Biology, Niigata University Graduate School of Medical and Dental Sciences, Niigata, Japan. ${ }^{18}$ Brain Research Institute, Niigata University, Niigata, Japan. ${ }^{19}$ Department of Neurology, Graduate School of Medicine, The University of Tokyo, Tokyo, Japan.
\end{abstract}

Cerebral small vessel disease (CSVD) causes dementia and gait disturbance due to arteriopathy. Cerebral autosomal recessive arteriopathy with subcortical infarcts and leukoencephalopathy (CARASIL) is a hereditary form of CSVD caused by loss of high-temperature requirement A1 (HTRA1) serine protease activity. In CARASIL, arteriopathy causes intimal thickening, smooth muscle cell (SMC) degeneration, elastic lamina splitting, and vasodilation. The molecular mechanisms were proposed to involve the accumulation of matrisome proteins as substrates or abnormalities in transforming growth factor $\beta$ (TCF- $\beta$ ) signaling. Here, we show that HTRA1 ${ }^{-1-}$ mice exhibited features of CARASIL-associated arteriopathy: intimal thickening, abnormal elastic lamina, and vasodilation. In addition, the mice exhibited reduced distensibility of the cerebral arteries and blood flow in the cerebral cortex. In the thickened intima, matrisome proteins, including the hub protein fibronectin (FN) and latent TCF- $\beta$ binding protein 4 (LTBP-4), which are substrates of HTRA1, accumulated. Candesartan treatment alleviated matrisome protein accumulation and normalized the vascular distensibility and cerebral blood flow. Furthermore, candesartan reduced the mRNA expression of Fn1, Ltbp-4, and Adamts/2, which are involved in forming the extracellular matrix network. Our results indicate that these accumulated matrisome proteins may be potential therapeutic targets for arteriopathy in CARASIL.

\section{Introduction}

Cerebral small vessel disease (CSVD) is a sporadic and hereditary arteriopathy of the cerebral small vessels that results in dementia and gait disturbance (1). The molecular mechanism of CSVD differs from that of large vessel disease, but the precise mechanism is still unknown (2). Therefore, therapeutic strategies based on the molecular mechanism have not been developed for CSVD.

Cerebral autosomal recessive arteriopathy with subcortical infarcts and leukoencephalopathy (CARASIL) is a hereditary form

Conflict of interest: The authors have declared that no conflict of interest exists. Copyright: () 2021, American Society for Clinical Investigation.

Submitted: May 22, 2020; Accepted: October 1, 2021; Published: November 15, 2021

Reference information: J Clin Invest. 2021;131(22):e140555.

https://doi.org/10.1172/JCl140555. of CSVD caused by a loss of function of high-temperature requirement A1 (HTRA1), a serine protease $(3,4)$. CARASIL is characterized by leukoencephalopathy, multiple lacunas, and reduced cerebral blood flow without hypertension (5). The arteriopathy of CARASIL shows intimal thickening, degeneration of smooth muscle cells (SMCs), splitting of the internal elastic lamina, and dilatation of vessels, and thus resembles sporadic CSVD (6-8). Intimal thickening is widespread in intracranial cerebral arteries, including large arteries as well as pial arteries and arterioles $(9,10)$. In contrast, degeneration of SMCs is most prominent in arterioles (9). It is unclear which morphological changes play a pivotal role in the pathogenesis of CARASIL.

Arteriopathy in CARASIL has been suggested to be caused by the accumulation of matrisome proteins, which are components of the extracellular matrix (ECM) (11-13). Matrisome proteins 
are composed of ECM proteins (core-matrisome proteins) and ECM-modifying proteins (matrisome-associated proteins; refs. $14,15)$. Indeed, matrisome proteins accumulate in the thickened intima in individuals with CARASIL $(3,13,16)$. Some of these accumulated matrisome proteins, including fibronectin ( $\mathrm{FN})$, are substrates for HTRA1 $(16,17)$.

Another hypothesis has been proposed regarding changes in transforming growth factor $\beta$ (TGF- $\beta$ ) signaling. HTRA1 overexpression suppresses TGF- $\beta$ signaling in cultured cells $(3,18,19)$. HTRA1 cleaves pro-TGF- $\beta 1$ (16). TGF- $\beta 1$ and proteins induced by TGF- $\beta$ signaling accumulate in the cerebral small vessels of patients with CARASIL, suggesting that TGF- $\beta$ signaling is enhanced in CARASIL $(3,16)$. On the other hand, decreased TGF- $\beta$ signaling has also been proposed (20). HTRA1 digests latent TGF- $\beta$-binding protein 1 (LTBP-1), which anchors inactive TGF- $\beta 1$ to the ECM (20). Decreased TGF- $\beta$ signaling has been demonstrated in cultured fibroblasts from HTRA1 ${ }^{-/-}$mice (20). Decreased TGF- $\beta$ signaling may induce degeneration of SMCs (21). Therefore, it remains unclear how TGF- $\beta$ signaling contributes to arteriopathy in CARASIL (20, 22-25).

If perturbation of the matrisome in cerebral small vessels or an increase in TGF- $\beta$ signaling plays an important role in developing arteriopathy in CARASIL, we hypothesized that angiotensin II type 1 receptor antagonists (AT1RBs) may be a candidate for treatment of arteriopathy. AT1RBs, including candesartan cilexetil, attenuate arteriopathy in several other organs by normalizing the perturbed matrisome (26). Candesartan suppresses FN expression (27) and inhibits TGF- $\beta$ signaling in the brain (28-30). In the present study, we aimed to elucidate the molecular mechanism of arteriopathy in CARASIL by examining whether candesartan cilexetil improves arteriopathy in $\mathrm{HTRA}^{-/-}$mice.

\section{Results}

HTRA1 is expressed in the endothelial cells of the pial arteries. The loss of HTRA1 expression in HTRA1 $1^{-/-}$mice was confirmed by RT-PCR (Supplemental Figure 1, A-C; supplemental material available online with this article; https://doi.org/10.1172/JCI140555DS1). HTRA1 is a secretory protein. Thus, we investigated the distribution of Htral mRNA by performing in situ hybridization to detect cells expressing HTRA1 (Supplemental Figure 1D). In HTRA1 ${ }^{+/+}$mice, Htra1 mRNA was expressed in astrocytes but not in neurons or microglia (Supplemental Figure 2A) (24). In pial arteries, Htra1 mRNA was expressed in endothelial cells (Supplemental Figure 2B), while in arterioles and capillaries, few endothelial cells expressed Htra1 mRNA (Supplemental Figure 2, C and D). Htra1 mRNA was not expressed in SMCs or pericytes (Supplemental Figure 2, B and E). Htra1 mRNA expression in astrocytes and endothelial cells was confirmed in cultured primary cells from $\mathrm{HTRA}^{+/+}$mice (Supplemental Figure 2, F and G).

Accumulation of matrisome proteins in the pial arteries and arterioles of HTRA1-/- mice and patients with CARASIL. HTRA1 $1^{-/-}$mice showed normal blood pressure and normal blood glucose levels (Supplemental Figure 3, A and B). By 24 months of age, HTRA1-/mice displayed no motor deficits, white matter lesions, or ischemic lesions compared with HTRA1 ${ }^{+/+}$mice (Supplemental Figure $3 \mathrm{C}$ ). There was also no difference in vascular density in the brain parenchyma between these 2 groups of mice (Supplemental Figure 3, D and E).
We conducted mass spectrometry (MS) analysis of the anterior and middle cerebral arteries of 24-month-old HTRA1 ${ }^{-/-}$and $\mathrm{HTRA}^{+/+}$mice because histochemical alterations are prevalent in the intracranial vessels of individuals with CARASIL (9). We identified 7381 proteins (Supplemental Table 1), and 416 showed significant changes in abundance between the 2 groups (increased proteins: 389, decreased proteins: 27; Supplemental Table 2). Gene Ontology (GO) enrichment analysis revealed that the increased proteins in $\mathrm{HTRA1}^{-/-}$mice were highly enriched into ECM components $\left(\mathrm{FDR}=5.67 \times 10^{-81} ;\right.$ Figure $1 \mathrm{~A}$ and Supplemental Table 5). One hundred fifty-five matrisome proteins were increased (Figure $1 \mathrm{~B}$ and Supplemental Table 3). The decreased proteins (including 4 matrisome proteins) were not significantly enriched in any GO term.

We performed topological analysis of protein-protein interaction networks with the proteomic data set and compared the following indexes to explore hubs in the network: closeness centrality $(\mathrm{Cc})$, betweenness centrality $\left(\mathrm{C}_{\mathrm{B}}\right)$, and degree of connectivity (D) $(31,32)$. We found that FN was the most critical hub, with the highest index scores in both networks composed of all changed proteins $\left(\mathrm{Cc}=0.49, \mathrm{C}_{\mathrm{B}}=0.18, \mathrm{D}=93\right)$ (Supplemental Table 2) and networks composed of changed matrisome proteins $(\mathrm{Cc}=0.59$, $\mathrm{C}_{\mathrm{B}}=0.37, \mathrm{D}=60$; Figure 1, C and D and Supplemental Table 3).

Next, we compared our results with data on matrisome proteins that Zellner et al. found to be changed in the brain parenchymal small vessels of HTRA1 ${ }^{-/-}$mice (13). We identified 42 increased matrisome proteins and 1 decreased matrisome protein that overlapped in both data sets (Supplemental Figure 4, A and B and Supplemental Table 4). Among them, we further analyzed the 6 most abundant proteins that were increased more than 2-fold in HTRA1 ${ }^{-/}$mice (FN, LTBP-1, LTBP-4, elastin, fibulin-5, and metalloproteinase inhibitor 3 [TIMP3]) by immunoblotting of cerebral blood vessels (Supplemental Table 4). Significant increases in the levels of FN, LTBP-4, elastin, TIMP3, and fibulin-5 (Figure 2A), but not LTBP-1 ( $P=0.064$; Supplemental Figure $5 \mathrm{~A})$, in HTRA1 $^{-/-}$mice were confirmed. The cellular type of FN (EDA ${ }^{+}$ $\mathrm{FN}$ ) was increased (Figure 2A), while the plasma type of FN was not increased in blood plasma (Supplemental Figure 5B). See complete unedited blots in the supplemental material.

Next, we assessed FN, LTBP-4, fibulin-5, and elastin by immunohistochemical analysis. As reported by Monet-Lepretre et al., we could not find a suitable antibody for detecting mouse TIMP3 by immunohistochemical analysis (33). FN, LTBP-4, and fibulin-5 markedly accumulated in the intima of large cerebral arteries, pial arteries, and arterioles but not in capillaries of HTRA1 $^{-/-}$mice (Figure 2, B-D and Supplemental Figure 5, C-H). Immunohistochemical analysis and immunoelectron microscopy revealed that FN, LTBP-4, and fibulin-5 accumulated in the subendothelial layer of the thickened intima of pial arteries (Supplemental Figure 6, A-E) and arterioles (Supplemental Figure $6, \mathrm{~F}$ and $\mathrm{G}$ ). Accumulation was also occasionally observed in the tunica media (Supplemental Figure 6H). We also found double-layered elastic lamina formation in the intima of pial arteries of $\mathrm{HTRA}^{-/-}$mice (Figure 2E). The elastic lamina on the luminal side was not uniform in thickness and was partially fragmented and colocalized with LTBP-4 (Figure 2E).

In patients with CARASIL, the accumulation of FN, LTBP-4, TIMP3, and fibulin-5 was confirmed in the pial arteries and the 
A

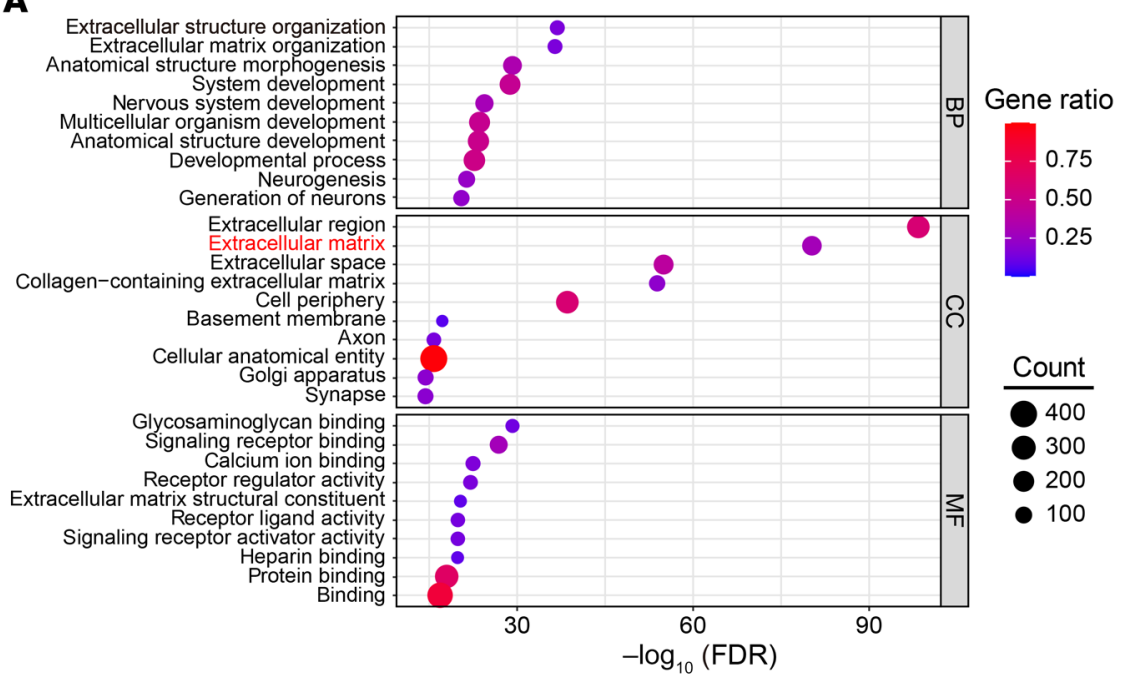

B

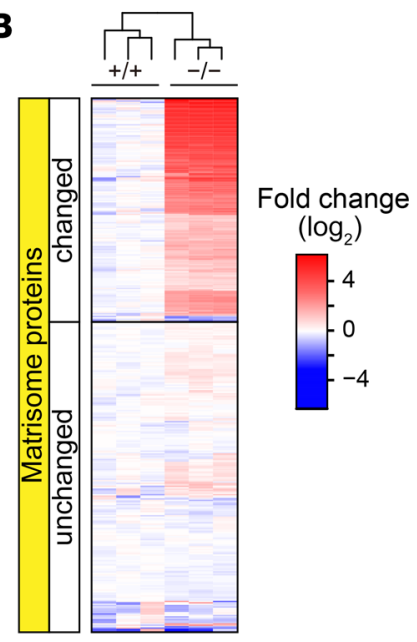

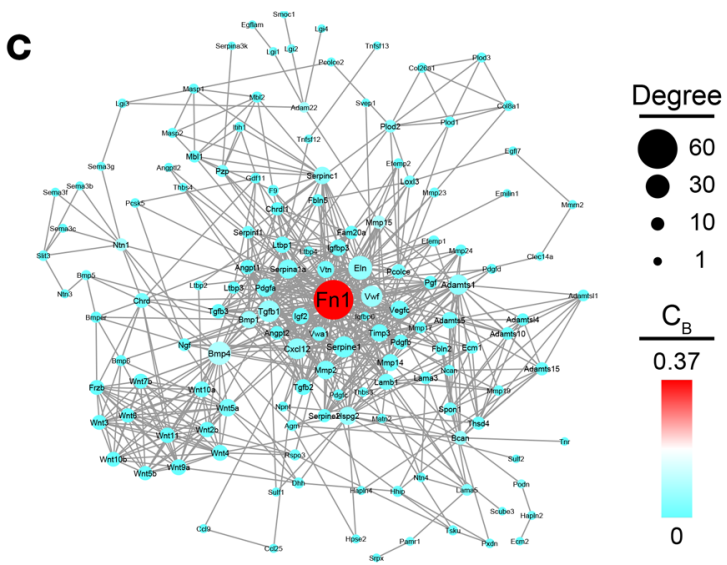

D

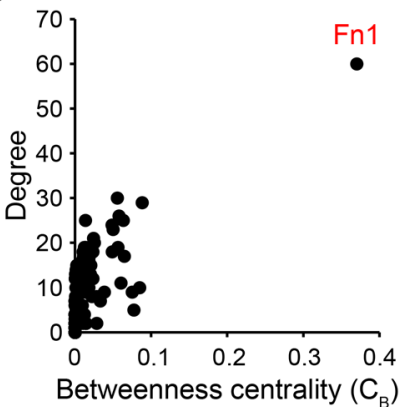

Figure 1. Matrisome protein accumulation with FN as a central hub in cerebral vessels in HTRA1-/- mice. (A) GO enrichment analysis of changed proteins in the cerebral arteries of HTRA $1^{-1-}$ mice. The plots are size-scaled by the number of changed proteins enriched for each $\mathrm{GO}$ term and color-scaled by gene ratio (ratio of the number of changed proteins to the number of proteins associated with a GO term). BP: biological process, CC: cellular component, MF: molecular function. (B) Heatmap of proteome data showing the abundance of matrisome proteins expressed as the log ${ }_{2}$ ratio of the average abundance in HTRA $1^{+/+}$mice ( $n=3$ animals per group). The upper section (changed) shows data for proteins with an adjusted $P<0.05$ and a fold change in protein abundance $>1.5$ or $<1.5^{-1}$. The lower section (unchanged) shows data for proteins that did not reach the set thresholds. (C) Protein-protein interaction network composed of fluctuating matrisome proteins. The nodes are size-scaled by degree and color-scaled by betweenness centrality $\left(C_{B}\right)$. Proteins (nodes) without interactions (edges) are not shown. (D) Scatter plot of the results of network topology analysis including degree and $\mathrm{C}_{\mathrm{B}}$ revealing $\mathrm{FN}$ (Fn1) as a major hub in the network.

arterioles but not in the capillaries (Figure 3, A-X and Supplemental Figure 7, A-D). FN, LTBP-4, and fibulin-5 were found in the thickened intima, while TIMP3 was found in the intima but was preferentially detected in the tunica media. In some vessels, LTBP- 4 and fibulin-5 displayed multilayered deposits (Figure 3, $\mathrm{H}$ and $\mathrm{W}$ ). Notably, LTBP-4 and fibulin-5 were frequently observed on the luminal side of multilayered elastin (Figure 3, $\mathrm{Y}$ and $\mathrm{Z}$ ).

We investigated whether HTRA1 digests LTBP-4 and elastin since FN, TIMP3, and fibulin-5 have been reported to be substrates of HTRA1 $(13,17,34)$. HTRA1 digested LTBP-4 at the same concentration at which it digested FN (Supplemental Figure 8A). However, elastin was not digested by HTRA1 even at a dose that was more than enough to degrade FN and LTBP-4 (Supplemental Figure 8A).

Because of the potential variability of TGF- $\beta$ signals in CARASIL, we analyzed the accumulation of TGF- $\beta$ and alterations in TGF- $\beta$ signaling in $\mathrm{HTRA1}^{-/-}$mice. Consistent with the MS analy- sis, immunoblotting analysis revealed increased levels of TGF- $\beta 1$ (latency-associated peptide for TGF- $\beta 1$ [LAP- $\beta 1]$ ) (Supplemental Figure 8B). A significant increase in TGF- $\beta 2$ levels, though lower than that in TGF- $\beta 1$ levels, was also observed (Supplemental Figure $8 \mathrm{~B})$. TGF- $\beta 1$ was detected in the intima of the pial arteries and the arterioles but not in the capillaries at 24 months of age (Supplemental Figure 8, C-E). However, immunohistochemical and immunoblotting analysis of the cerebral arteries and the cortices of HTRA1 $1^{--}$mice did not reveal any changes in phosphorylated SMAD (pSMAD) levels (Supplemental Figure 8, F-J). We also did not find any changes in noncanonical SMAD-independent TGF- $\beta$ signaling (Supplemental Figure 9, A-C) or in the expression levels of TGF- $\beta$-regulated genes (Supplemental Figure 9D).

Candesartan ameliorates the accumulation of matrisome proteins and prevents vascular remodeling. AT1RBs, including candesartan cilexetil, have been reported to reduce matrisome protein accumu- 
A
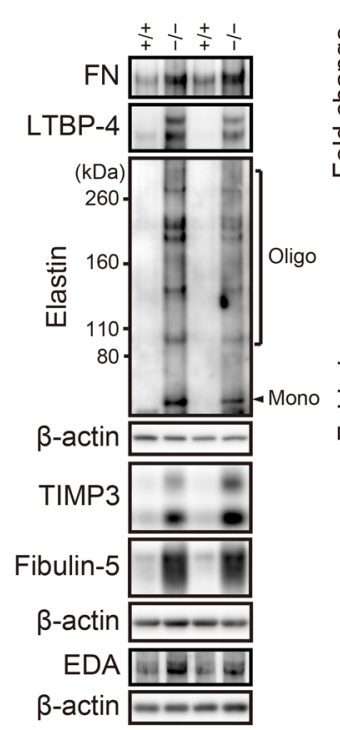

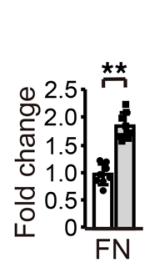

口 $\mathrm{HTRA} 1^{+/+}$

HTRA1 ${ }^{-1-}$

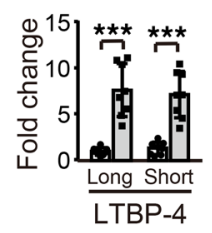

B

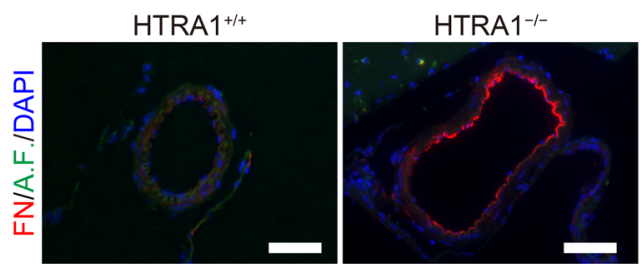

C

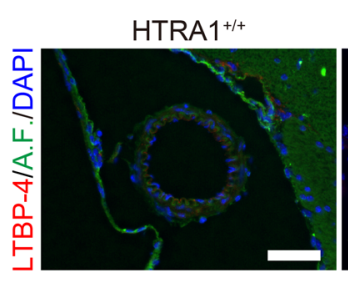

D

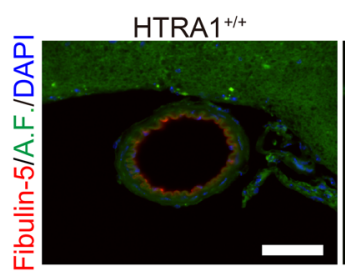

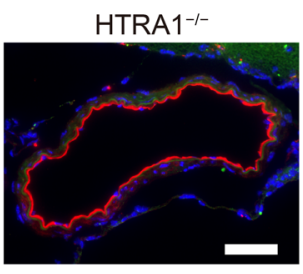

HTRA $1^{-1-}$

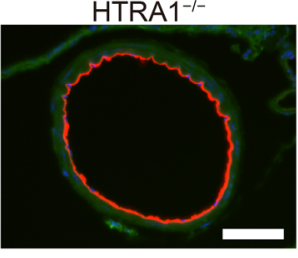

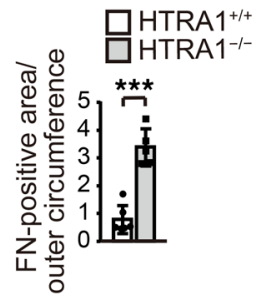
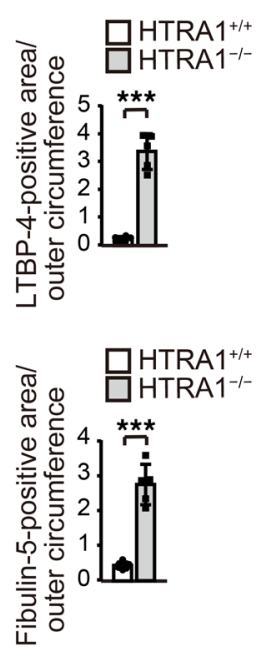

E

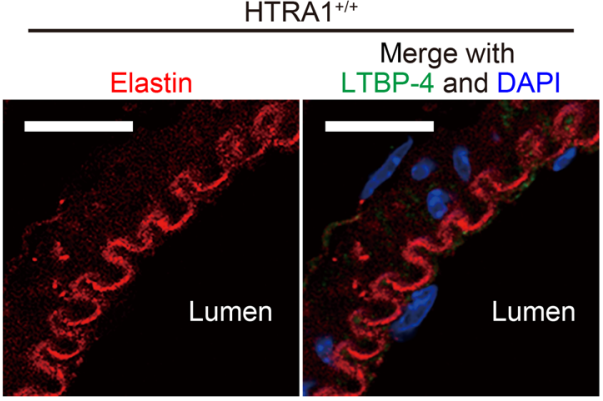

HTRA $1^{-1-}$

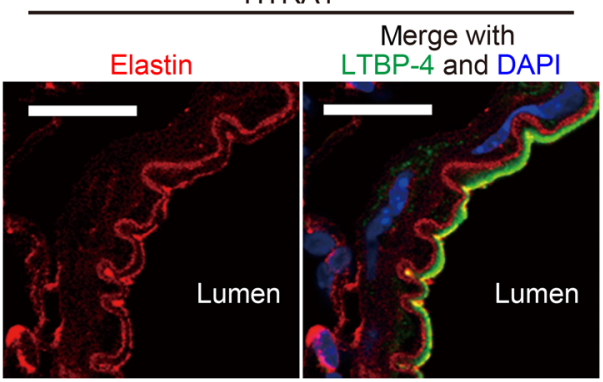

Figure 2. Matrisome protein accumulation with ectopic elastic lamina formation in the intima of the cerebral arteries of HTRA1/- mice. (A) Matrisome protein levels in the anterior and middle cerebral arteries were assessed by immunoblotting ( $n=5-8$ animals per group). The samples used for TIMP3 and fibulin- 5 were identical and distinct from those used for the other matrisome protein quantifications. The bar graphs show values relative to those for $\mathrm{HTRA}^{+/+}$mice. (B-D) Immunohistochemical detection of FN (B), LTBP-4 (C), and fibulin-5 (D) in the anterior cerebral arteries at 24 months of age. Autofluorescence, A.F. Scale bars $=50 \mu \mathrm{m}$. Bar graphs show the quantification of FN-, LTBP-4-, and fibulin-5-immunopositive areas. The FN-, LTBP-4-, and fibulin-5-positive areas in the vessel wall in each image were quantified and normalized to the outer circumference of the vessel to eliminate the effects of vascular dilation in HTRA1 ${ }^{-1-}$ mice ( $n=5-6$ animals per group). (E) Simultaneous immunohistochemical detection of elastin and LTBP-4 in the anterior cerebral arteries. Three mice per group were analyzed. Ectopic elastic lamina formation was observed in all HTRA1 ${ }^{-1-}$ mice. Typical images are shown. Scale bars $=10 \mu \mathrm{m}$. The data represent the mean \pm SD. ${ }^{* *} P<0.01$ and ${ }^{* *} P<0.001$ with 2-tailed unpaired $t$ test or the Mann-Whitney $U$ test $(\mathbf{A}-\mathbf{D})$.

lation in blood vessels independently of their hypotensive effect (26, 35-37). In addition, since candesartan suppresses FN expression $(38,39)$, we investigated whether candesartan shows a protective effect on matrisome protein accumulation in $\mathrm{HTRA1}^{-/-}$mice. In $\mathrm{HTRA1}^{-/-}$mice, the accumulation of FN and LTBP-4 began at 4 months of age and increased with age (Supplemental Figure 10, A and B). Therefore, we began administration of candesartan and the calcium-channel blocker amlodipine, a control with comparable antihypertensive effects, to HTRA1 ${ }^{-/-}$mice at 4 months of age and examined the effects of these drugs at 24 months of age (Supplemental Figure 11, A and B). Immunohistochemical analysis revealed that FN levels were substantially reduced and that the levels of
LTBP-4 and fibulin-5 were almost halved in candesartan-treated $\mathrm{HTRA1}^{--}$mice (Figure 4, A and B and Supplemental Figure 11, $\mathrm{C}-\mathrm{F})$. In contrast, amlodipine slightly reduced the FN levels but did not reduce the levels of LTBP-4 and fibulin-5 (Figure 4, A and B and Supplemental Figure 11, C-F). Immunoblotting analysis of the cerebral arteries of $\mathrm{HTRA1}^{-/-}$mice confirmed that FN, LTBP-4, $\mathrm{EDA}^{+} \mathrm{FN}$, and elastin levels were decreased in candesartan-treated $\mathrm{HTRA1}^{-/-}$mice (Figure $4 \mathrm{C}$ ). In addition, the accumulation of TIMP3, fibulin-5, and TGF- $\beta 1$ was attenuated by candesartan treatment (Supplemental Figure 11G). Concomitantly, the levels of pSMAD3 but not pSMAD5 or pErk1/2 were downregulated by candesartan treatment (Supplemental Figure 11G and ref. 40). 

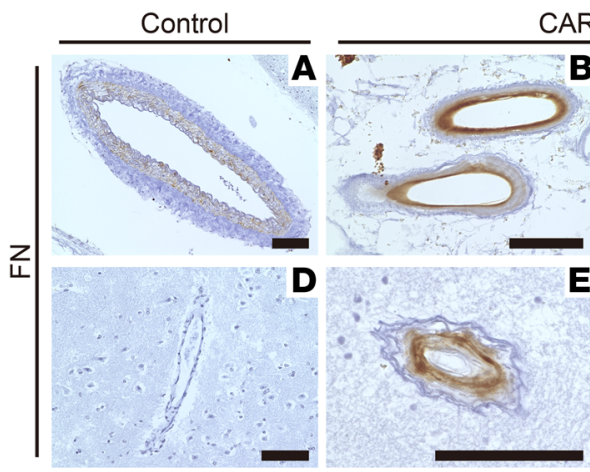

CARASIL

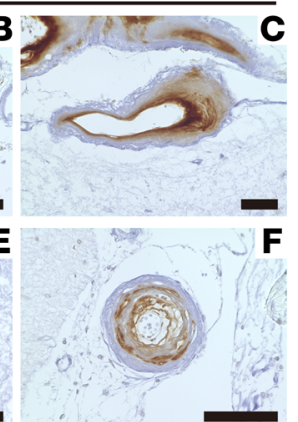

G
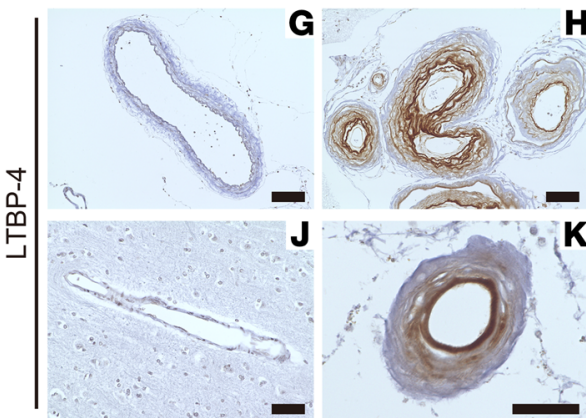

H

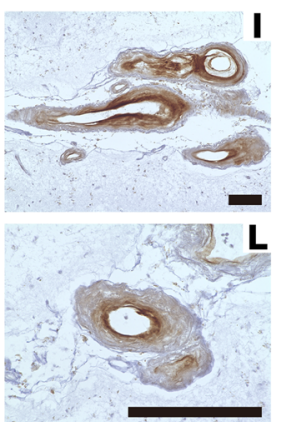

$\mathbf{Y}$
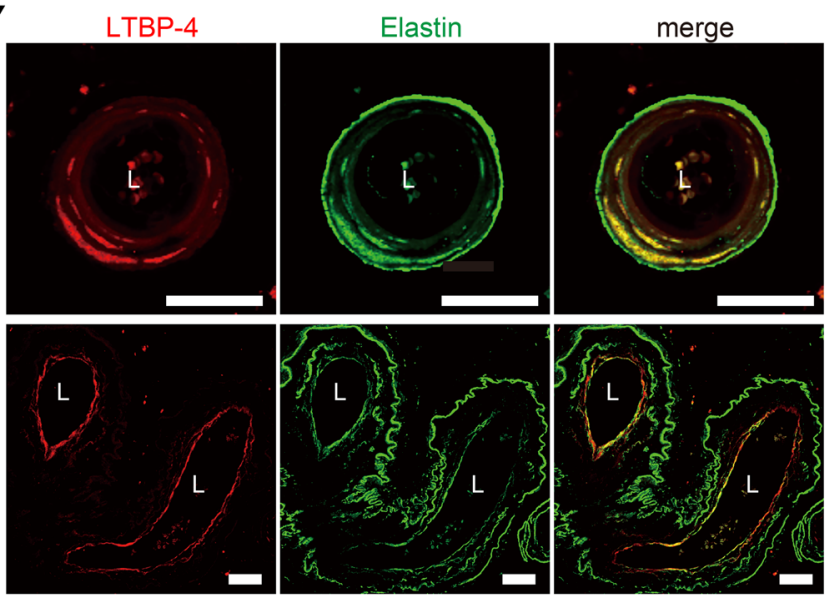
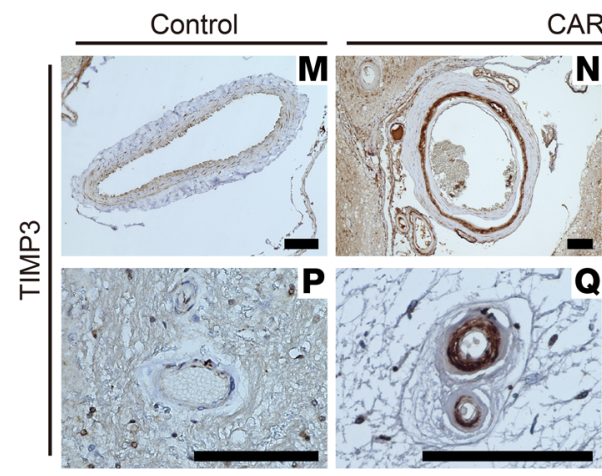

CARASIL
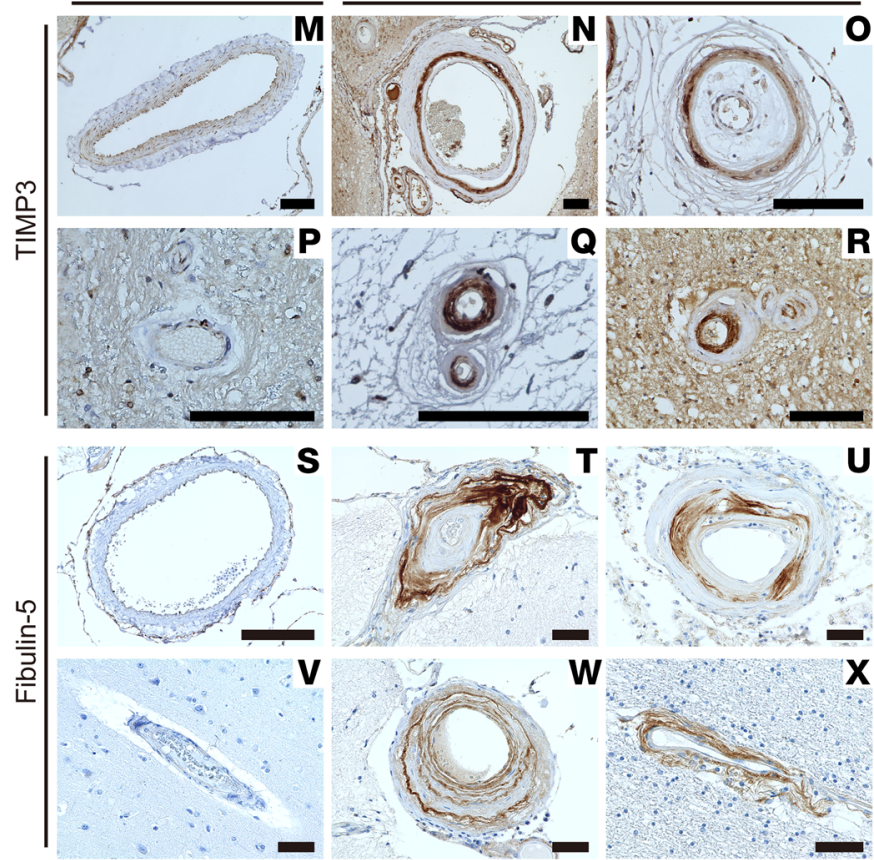

$\mathbf{Z}$
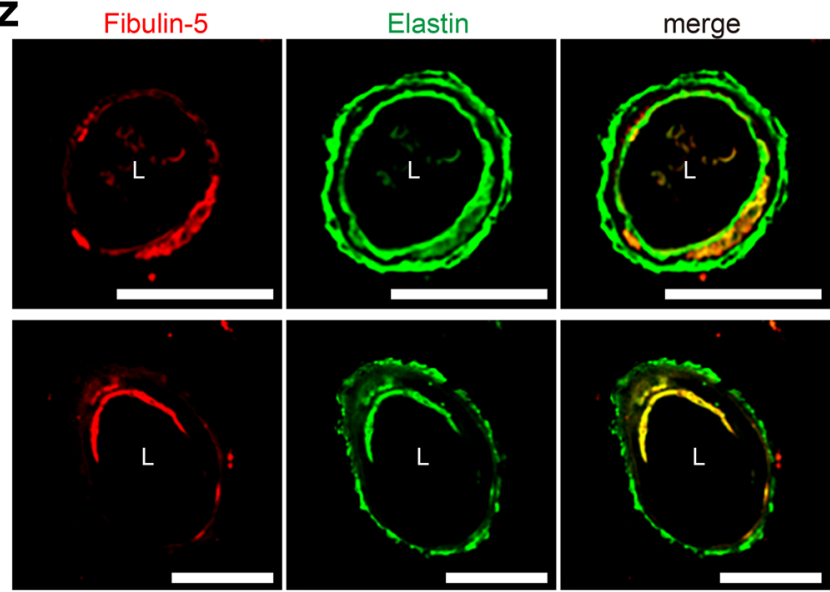

Figure 3. Accumulation of matrisome proteins and colocalization of elastic lamina and LTBP-4 or fibulin-5 in the cerebral vessels of patients with CARASIL. (A-X) FN (A-F), LTBP-4 (G-L), TIMP3 (M-R), and fibulin-5 (S-X) immunoreactivity in the cerebral arteries of controls (A, D, G, J, M, P, S, and V) and patients with CARASIL (B, C, E, F, H, I, K, L, N, O, Q, R, T, U, W, and X). Panels B, C, E, H, K, O, Q, and W were obtained from patient 1 (a 46-year-old woman), and panels $\mathbf{F}, \mathbf{I}, \mathbf{L}, \mathbf{N}, \mathbf{R}, \mathbf{T}, \mathbf{U}$, and $\mathbf{X}$ were obtained from patient 2 (a 54-year-old man); both patient 1 and patient 2 had a homozygous nonsense mutation in the HTRA1 gene (p.R302ter). Apparent accumulation of FN, LTBP-4, TIMP3, and fibulin-5 was detected in the cerebral vessels of patients with CARASIL. For comparison, autopsy samples from 4 control subjects (a 49-year-old woman, a 51-year-old woman, an 89-year-old man, and a 51-year-old man) were subjected to the same immunohistochemical analysis. No evident accumulation of these molecules was found in the cerebral arteries of the controls. Representative micrographs of pial (A-C, F-I, M-O, S-U, and $\mathbf{W})$ and parenchymal ( $\mathbf{D}, \mathbf{E}, \mathbf{J}, \mathbf{K}, \mathbf{L}, \mathbf{P}, \mathbf{Q}, \mathbf{R}, \mathbf{V}$, and $\mathbf{X})$ arteries are shown. Scale bars = $100 \mu \mathrm{m}$. (Y and Z) Simultaneous detection of elastin and LTBP-4 (Y) or fibulin-5 (Z) in cerebral arteries of a CARASIL patient (a 46-year-old woman). In the LTBP-4 or fibulin- 5 accumulation sites, colocalization with elastin was frequently found. Details of the patients with CARASIL and controls are shown in Supplemental Table 18. L: vascular lumen. Scale bars $=50 \mu \mathrm{m}$.

We further conducted MS analysis of the cerebral vessels of candesartan-treated and untreated 24-month-old HTRA1 ${ }^{-1}$ mice. We identified 6123 proteins, and 1107 showed significant changes in abundance in candesartan-treated HTRA1 ${ }^{-/-}$mice (increased proteins: 804, decreased proteins: 303; Supplemental Table 6). Among the 360 proteins that were increased in untreated $\mathrm{HTRA}^{-/-}$mice compared with untreated $\mathrm{HTRA1}^{+/+}$ mice and identified by 2 or more unique peptides in the second
MS analysis, $48.9 \%$ of the proteins were decreased by candesartan treatment (group 1; Supplemental Tables 2, 6, and 7). GO enrichment analysis of group 1 revealed notable enrichment into ECM components $\left(\mathrm{FDR}=1.03 \times 10^{-52}\right.$; Figure $4 \mathrm{D}$, Supplemental Figure 12A, and Supplemental Table 9). In contrast, among the proteins that were increased in untreated $\mathrm{HTRA}^{-/-}$mice compared with untreated $\mathrm{HTRA}^{+/+}$mice but not decreased by candesartan treatment (group 2), no such characteristic enrichment 
A
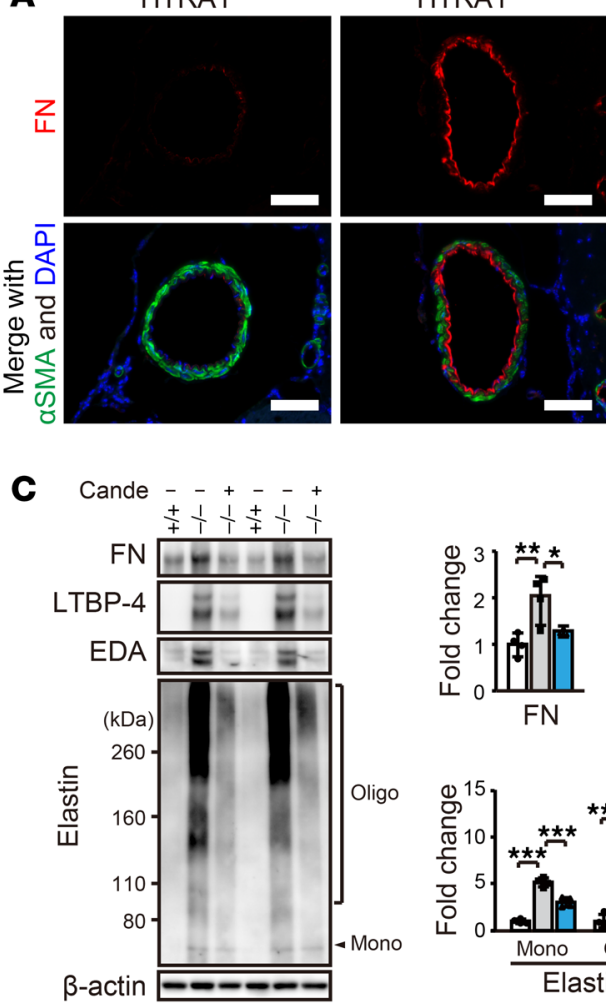

D

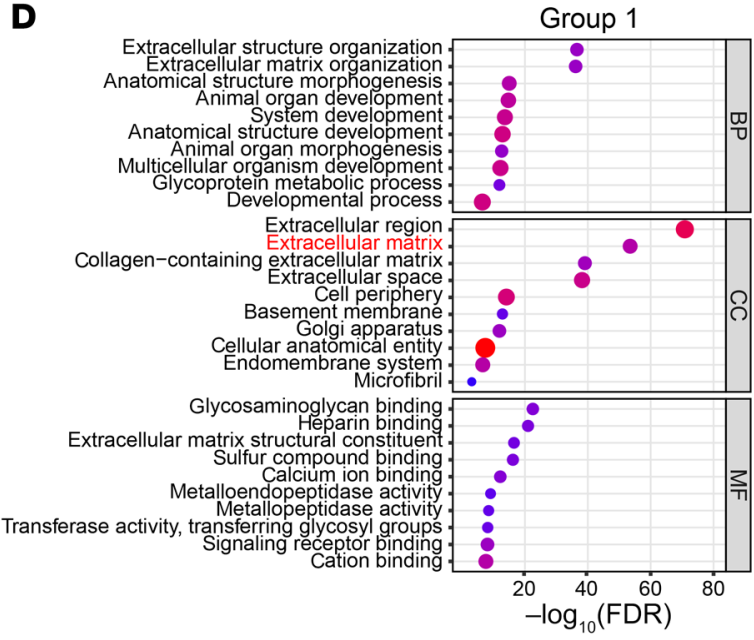

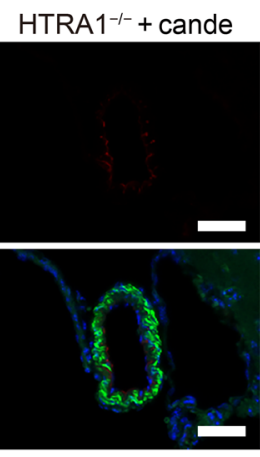

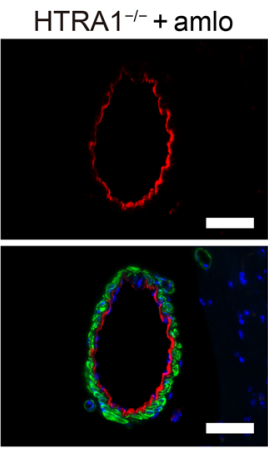

B
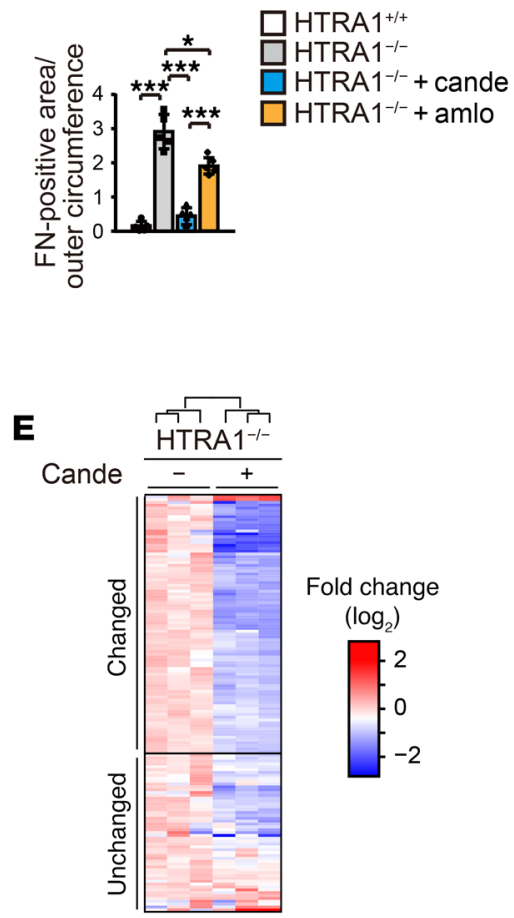
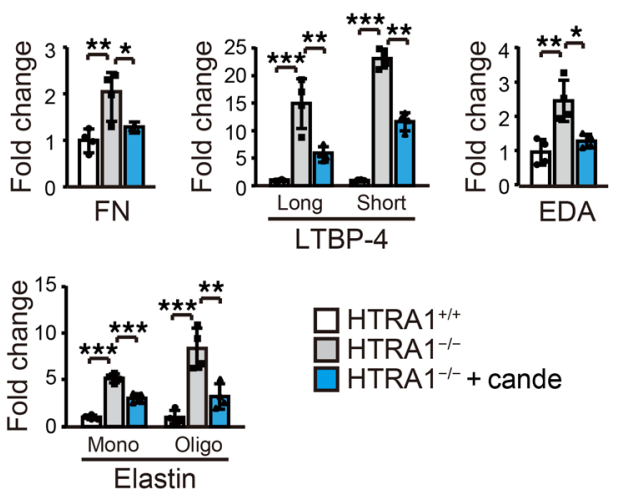

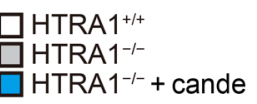

HTRA $1^{+/+}$

HTRA $1^{-1-}+$ cande

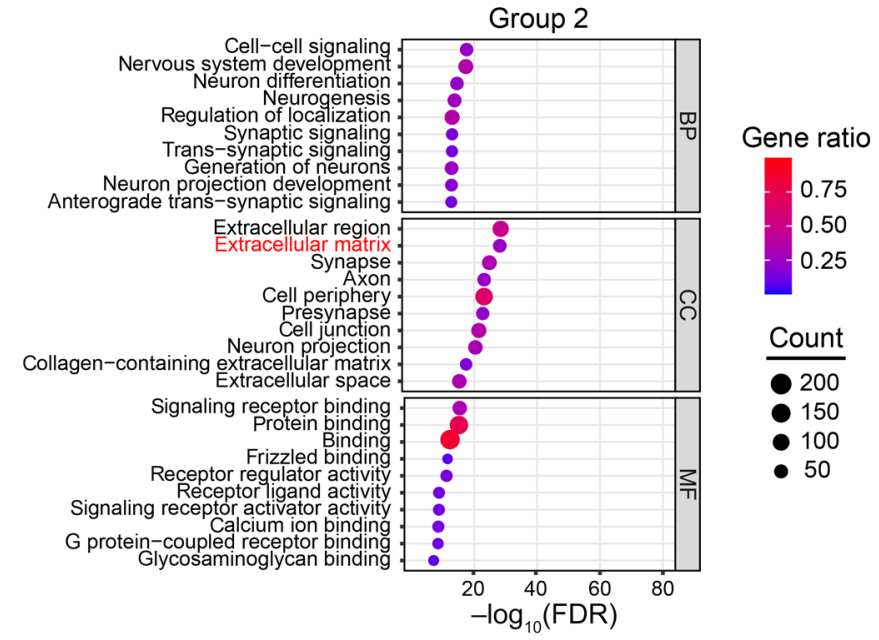

Figure 4. Candesartan treatment prevents matrisome protein accumulation in HTRA1 ${ }^{-/-}$mice. (A and B) FN was detected in the anterior cerebral arteries by immunohistochemical staining with medial visualization by aSMA immunostaining at 24 months of age (A). Scale bars $=50 \mu \mathrm{m}$. (B) The area positive for FN was quantified and normalized to the outer circumference ( $n=5-6$ animals per group). (C) Quantification of matrisome proteins in the cerebral arteries of 24-month-old $\mathrm{HTRA}^{+/+}$and $\mathrm{HTRA}^{-/-}$mice with or without candesartan treatment by immunoblotting analysis ( $n=4-5$ animals per group). The bar graphs show values relative to those for HTRA $1^{+/+}$mice. The data represent the mean \pm SD. ${ }^{*} P<0.05$, ${ }^{* *} P<0.01$, and ${ }^{* * *} P<0.001$ with Bonferroni's post hoc test (B and C). (D) GO enrichment analysis of proteome data for candesartan-treated and untreated HTRA1 ${ }^{-/-}$mice. Group 1 represents the protein set on which candesartan showed an inhibitory effect against increasing changes in HTRA1-/- mice, and group 2 represents the protein set for which no inhibitory effect of candesartan treatment was observed (Supplemental Figure 12A). (E) Heatmap of the data from the second MS analysis showing protein abundance in candesartan-treated and untreated $\mathrm{HTRA}^{1 /-}$ mice expressed as the $\log _{2}$ ratio of average abundance in untreated HTRA1 ${ }^{-/-}$mice ( $n=3$ animals per group). The presented proteins are matrisome proteins that were identified by the first MS analysis as being increased in HTRA ${ }^{1 /-}$ mice compared with $\mathrm{HTRA}^{+/+}$mice and identified by 2 or more unique peptides in the second MS analysis. Amlo, amlodipine.

into ECM components was observed (Figure 4D, Supplemental Tables 8 and 10). Indeed, of the 145 matrisome proteins that were increased in untreated HTRA1 ${ }^{-/-}$mice and identified by 2 or more unique peptides in the second MS analysis, 88 proteins
(60.7\%) were decreased by candesartan treatment (Figure $4 \mathrm{E}$ and Supplemental Table 11).

Immunohistochemical analysis of the pial arteries of 24month-old HTRA1 ${ }^{-/}$mice showed intimal thickening and lumi- 
A

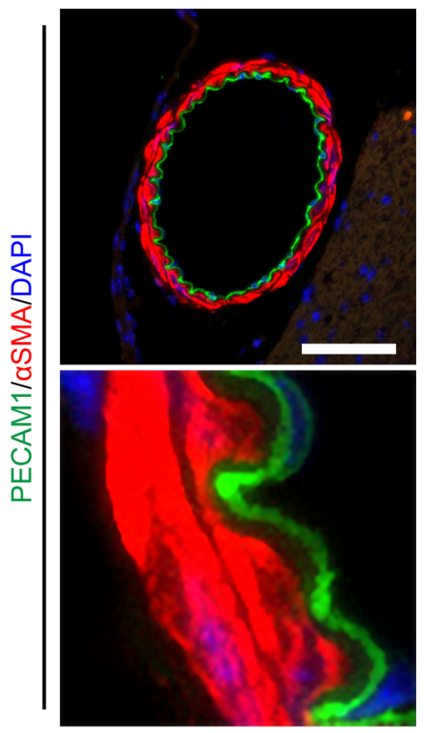

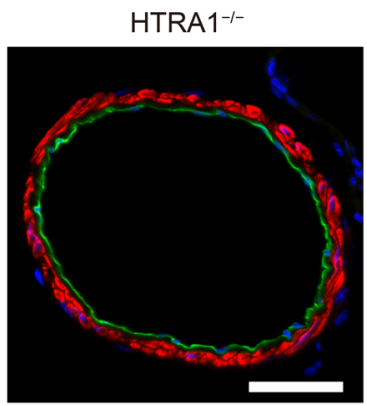
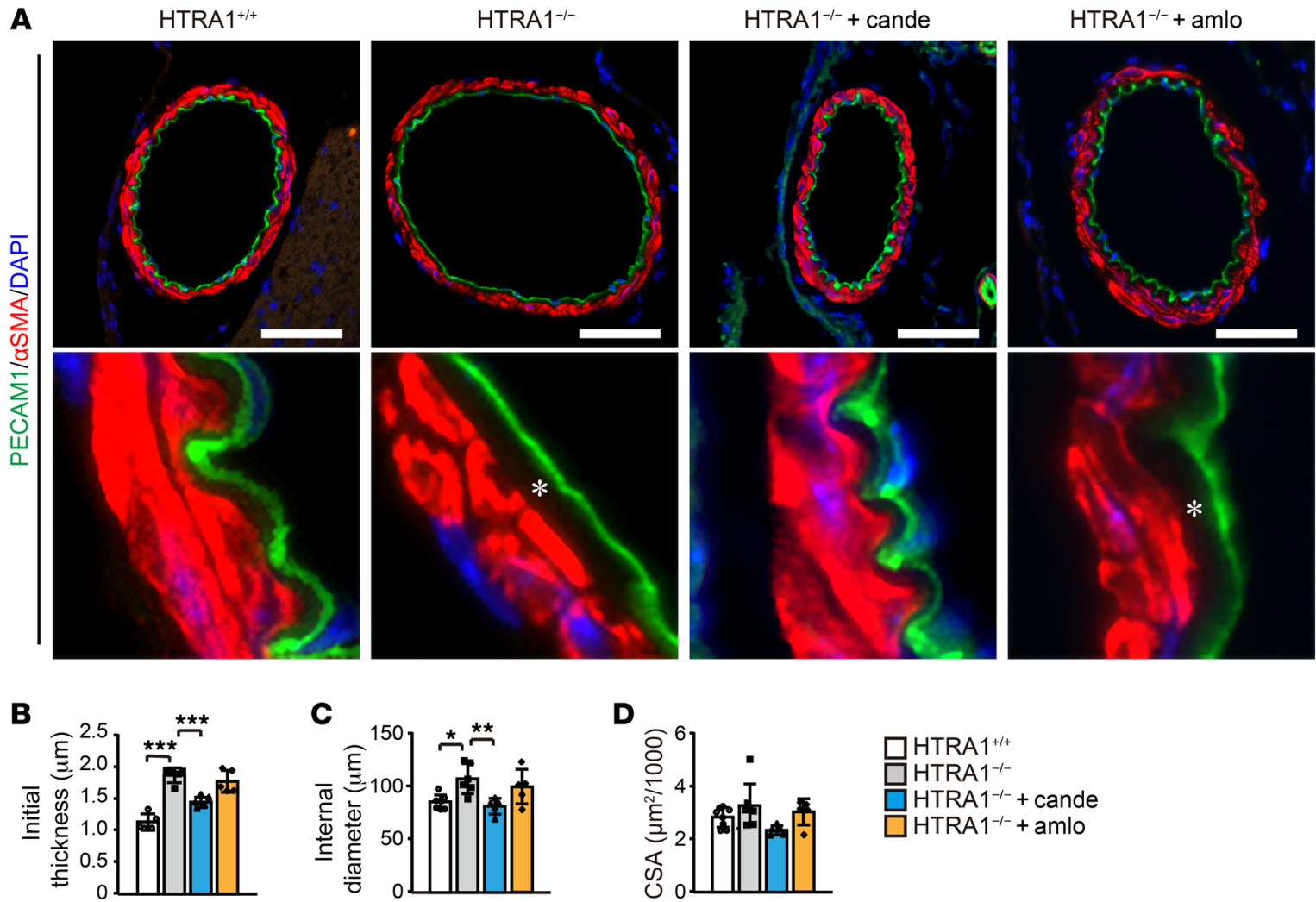

D
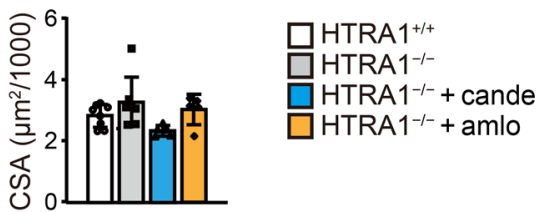

$\mathbf{E}$

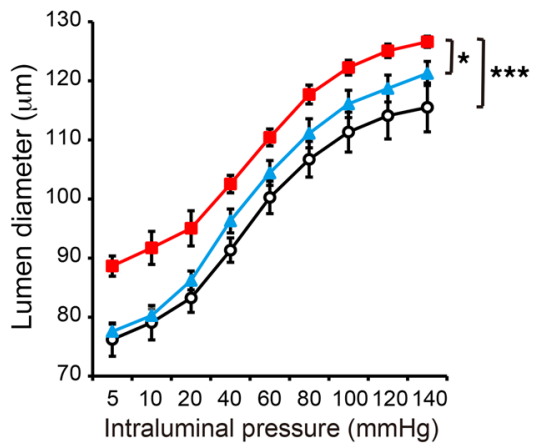

$\mathbf{F}$

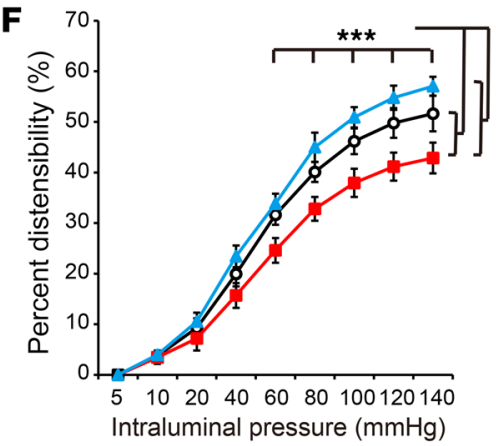

$\rightarrow-\mathrm{HTRA} 1^{+1+}$

$\rightarrow-\mathrm{HTRA} 1^{-1-}$

$-\mathrm{HTRA} 1^{-1-}+$ cande

Figure 5. Candesartan prevents cerebrovascular dilation and normalizes vascular distensibility in HTRA1 ${ }^{-/-}$mice. (A) Representative images of PECAM1and $\alpha S M A$-stained cross-sections of the anterior cerebral arteries of 24-month-old mice. Scale bars $=50 \mu \mathrm{m}$. The lower image is a high-magnification image of the upper micrograph. HTRA1/- mice showed increased subendothelial thickness from the inner edge of SMCs to the endothelial cell layer, in which ECM proteins accumulated (white asterisks). Intimal thickening was ameliorated by candesartan treatment but not amlodipine treatment. (B-D) The intimal thickness (B), internal diameter (C), and medial CSA (D) for $\mathrm{HTRA}^{+/+}$and $\mathrm{HTRA} 1^{-/-}$mice with or without candesartan or amlodipine treatment from 4 to 24 months of age were measured for each image and are presented. Five to 7 arterial sections per animal were analyzed ( $n=5-6$ animals per group). ${ }^{*} P<0.05$, ${ }^{* *} P<0.01$, and ${ }^{* *} P<0.001$ with Bonferroni's post hoc test (B and $\mathbf{C}$ ). (E) Middle cerebral arteries were pressurized ex vivo to 5 to 140 $\mathrm{mmHg}$, and their lumen diameter was tracked with a pressure myograph system under no-flow and calcium-free conditions. (F) Graph showing the passive distensibility versus intraluminal pressure of middle cerebral arteries of $\mathrm{HTRA}^{+/+}$and $\mathrm{HTRA} 1^{-/-}$mice with or without candesartan treatment from 4 to 24 months of age ( $n=5-6$ animals per group). Candesartan treatment attenuated structural and mechanical property changes in $\mathrm{HTRA1}^{-1-}$ mice. ${ }^{*} P<0.05$ and ${ }^{* *} P<0.001$ in $\mathbf{E}$ (significant difference between groups) with Bonferroni's post hoc test. ${ }^{* *} P<0.001$ in $\mathbf{F}$ (significant difference between groups at each indicated pressure level) with Bonferroni's post hoc test. The data represent the mean \pm SD.

nal dilatation resembling those observed in CARASIL patients (Figure 5, A-C). These alterations were improved by candesartan treatment but not amlodipine treatment (Figure 5, A-C). On the other hand, the cross-sectional areas (CSAs) of the tunica media and the number of SMCs were not different (Figure 5, A and D and Supplemental Figure 12B). Even in the arterioles, where CARASIL induced the most prominent degeneration of the tunica media, there was no change in staining for $\alpha$ smooth muscle actin ( $\alpha$ SMA; Supplemental Figure $12 \mathrm{C}$ and ref. 9). At 16 months of age, there was no increase in lumen diameter (Supplemental Figure 12D).

We further analyzed the lumen diameter under physiological pressure loading using the middle cerebral artery. An increase in vessel diameter in $\mathrm{HTRA}^{-/-}$mice was also demonstrated under physiological pressure loading (Figure $5 \mathrm{E}$ ). In addition, the cere- 
A

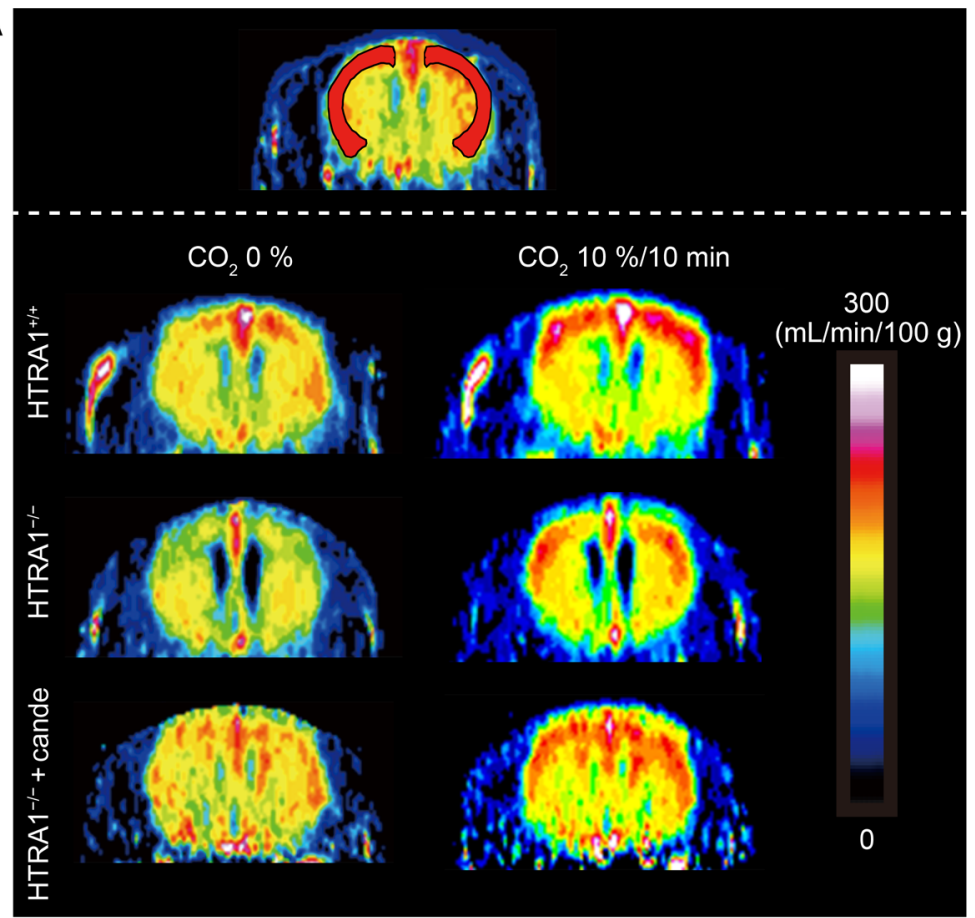

$\square \mathrm{HTRA} 1^{+/+}$

HTRA1-1-

HTRA $1^{-1-}+$ cande

B

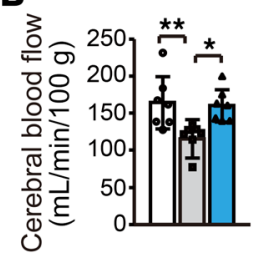

C

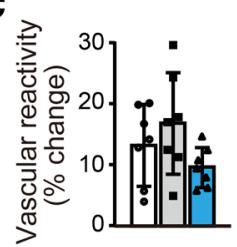

Figure 6. Candesartan prevents cerebral hypoperfusion in HTRA1 ${ }^{-/-}$ mice. (A-C) Resting cerebral blood flow and response of cerebral blood flow to hypercapnia ( $10 \% \mathrm{CO}_{2}$ inhalation) in $\mathrm{HTRA}^{+/+}$and $\mathrm{HTRA}^{-/-}$mice with or without candesartan treatment measured by CASL using MRI at 16 to 20 months of age ( $n=7$ animals per group). (A and $\mathbf{B}$ ) Resting cerebral blood flow in the cerebral cortex was calculated from regions of interest in the bilateral hemisphere, as indicated by red regions in (A). (A and C) Cerebral blood flow increase (\%) in the cerebral cortex in response to hypercapnia. Cerebral blood flow during $10 \% \mathrm{CO}_{2}$ inhalation was measured following measurement of resting cerebral blood flow. The data represent the mean \pm SD. ${ }^{*} P<0.05$ and ${ }^{*} P<0.01$ with Bonferroni's post hoc test (B). bral vessels of $\mathrm{HTRA1}^{-/-}$mice showed reduced distensibility (Figure $5 \mathrm{~F}$ ). Candesartan ameliorated vascular enlargement and normalized vascular distensibility (Figure 5, E and F).

Candesartan prevents a decrease in cerebral blood flow in HTRA1 ${ }^{-/}$mice. We examined cerebral blood flow in HTRA1 ${ }^{-/-}$ mice from 16-20 months of age using magnetic resonance imaging (MRI). The resting cerebral blood flow was significantly reduced in the cerebral cortex but not in the striatum of HTRA1 ${ }^{-1}$ mice (Figure 6, A and B, Supplemental Figure 12E, refs. 41-43). Notably, candesartan administration normalized cerebral blood flow in HTRA1 ${ }^{-/-}$mice (Figure 6, A and B). In the cerebral cortex, the vascular response to hypercapnia ( $10 \% \mathrm{CO}_{2}$ inhalation), a known cerebral vasodilator, did not differ between $\mathrm{HTRA}^{-/-}$mice and $\mathrm{HTRA}^{+/+}$mice (Figure 6, A and C).

Candesartan fails to prevent alterations in SMCs and pericytes. To explore the involvement of SMCs in the improvements in cerebral blood flow and vascular distensibility by candesartan treatment, we first focused on the expression of genes encoding SMC contractile proteins in the cerebral arteries of $\mathrm{HTRA}^{-/-}$mice. The expression levels of the genes significantly decreased after 12 months of age (Figure 7A). However, treatment with candesartan had no effect on the expression of these mRNAs (Figure 7B, and Myh11 in Supplemental Figure 13B).

Regarding pericytes, electron microscopy revealed that pericytes were significantly detached from endothelial cells in $\mathrm{HTRA}^{-/-}$mice (Figure 7C). The pericyte coverage rate of the capillary wall was also significantly reduced in $\mathrm{HTRA}^{-/-}$mice (Figure 7D). However, treatment with candesartan did not improve the pericyte coverage rate (Figure 7D). Blood-brain barrier function was preserved in $\mathrm{HTRA}^{-/-}$mice (Supplemental Figure 12, $\mathrm{F}$ and $\mathrm{G}$ and ref. 44 ).

Candesartan decreases the expression of Adamtsl2, Fn1, Ltbp-4, and Fbln5. To explore the molecular pathways affected by can- desartan, we performed RNA sequencing (RNA-seq) analysis of the anterior and middle cerebral arteries of HTRA $1^{+/+}$mice and HTRA1 $^{-/-}$mice with or without candesartan treatment at 4 and 24 months of age. Candesartan was administered for a short (1 week at 4 months of age) or long (from 4 to 24 months of age) period. First, we identified the genes with expression levels that were altered by HTRA1 deficiency. We identified 12 and 11 differentially expressed genes at 4 and 24 months of age, respectively. There were, however, no overlapping genes between the groups (Supplemental Figure 13, A and B, and Supplemental Tables 12 and 13). The changes in the expression of only 4 and 6 of these genes were reproduced by droplet digital PCR (ddPCR) analysis (Supplemental Figure 13, A and B and ref. 45). Next, we investigated the effect of candesartan on gene expression. Among the genes with altered expression levels in $\mathrm{HTRA}^{-/-}$mice compared with $\mathrm{HTRA1}^{+/+}$ mice, only Fblim1 was affected by candesartan treatment (Supplemental Figure 13A). As compared with gene expression levels of candesartan-treated and untreated $\mathrm{HTRA}^{-/-}$mice, we identified 10 and 84 genes that were differentially expressed in short-term and long-term candesartan-treated $\mathrm{HTRA}^{-/-}$mice, respectively (Figure 8, A and B and Supplemental Tables 14 and 15). Adamt$s l 2$, which encodes a matrisome-associated protein involved in FN network formation, was the only gene that overlapped between the groups (Figure 8, A-C and refs. 46, 47). ddPCR analysis showed that short-term treatment with amlodipine also slightly reduced Adamtsl2 expression, whereas candesartan treatment more highly reduced Adamtsl2 expression (Figure 8, D and E). Adamtsl2 mRNA expression was decreased by $69.5 \%$ and $49.0 \%$ in short-term and long-term candesartan-treated HTRA1 ${ }^{-/-}$mice, respectively, compared with untreated $\mathrm{HTRA} 1^{-/-}$mice. In addition, ddPCR analysis revealed that candesartan but not amlodipine exerted inhibitory effects on $F n 1, E D A^{+} F n 1, L t b p-4$, and $F b l n 5$ mRNA expression (Figure 8F) and that Timp3 mRNA expression was downregulat- 
A

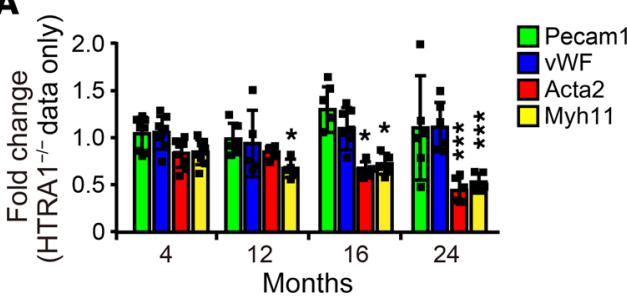

B

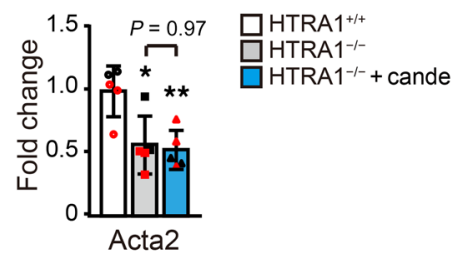

\section{C}

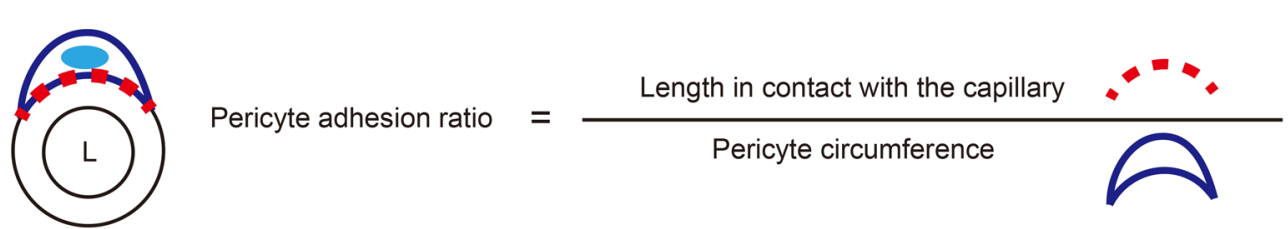

HTRA $^{+/+}$ HTRA $^{-1-}$
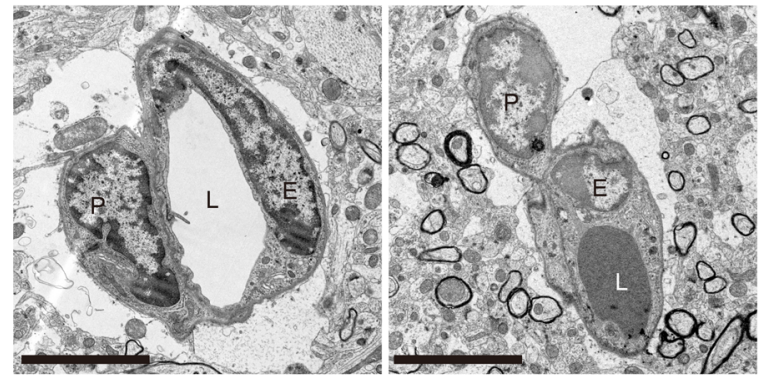

$\square \mathrm{HTRA}^{+/+}$
$\square \mathrm{HTRA} 1^{-1-}$

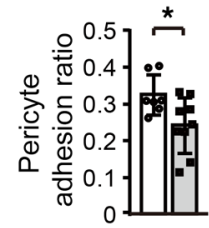

D
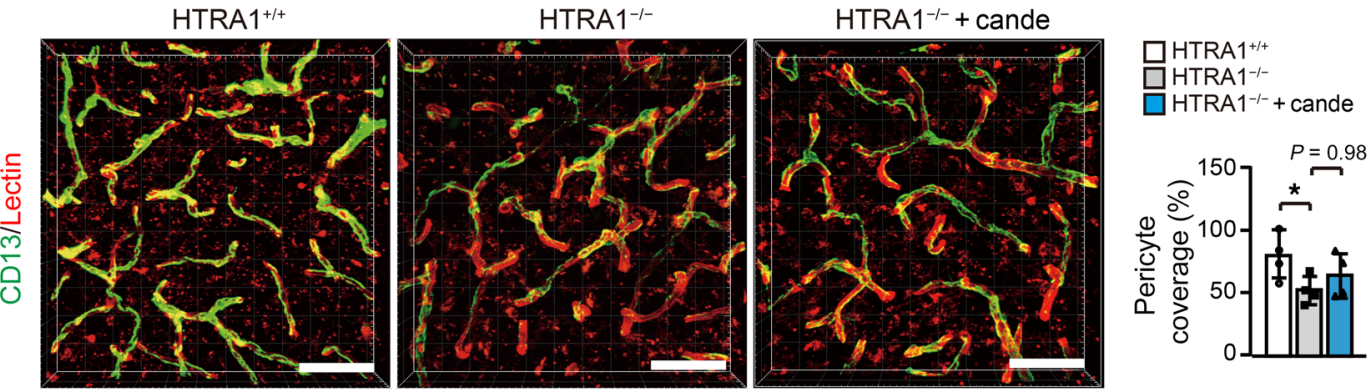

Figure 7. Candesartan fails to prevent mural cell alterations in HTRA1-/- mice. (A) Quantification of the expression of contractile SMC markers in the anterior and middle cerebral arteries at $4,12,16$, and 24 months of age. Only HTRA1 $1^{-/-}$mouse data are shown as a ratio relative to the HTRA ${ }^{+/+}$mice $(n=$ 5-8 animals per group). (B) The effect of candesartan treatment on the reduction in contractile SMC gene expression in HTRA1 ${ }^{-1-}$ mice. Expression levels relative to those in $\mathrm{HTRA}^{+/+}$mice are presented ( $n=5$ animals per group). (C) The pericyte-capillary adhesion ratio was estimated by analysis of electron micrographs. The upper panels show the formula used to determine the pericyte adhesion ratio. The pericyte adhesion ratio is the ratio of the length of the adhesion region between a pericyte and a capillary to the perimeter of the pericyte. The lower images are representative electron microscopic images of capillaries of $\mathrm{HTRA}^{+/+}$and HTRA1 ${ }^{-/-}$mice at 24 months of age and include endothelial cells and pericytes. The bar graph shows the pericyte adhesion ratio ( $n=7-9$ animals per group). Scale bars $=5 \mu \mathrm{m}$. L: lumen; P: pericyte; E: endothelial cell. (D) Representative 3D reconstructed images of capillaries in the cerebral cortices of 24-month-old $\mathrm{HTRA}^{+/+}$and $\mathrm{HTRA1}^{-/-}$mice with or without candesartan treatment. CD13-immunopositive pericytes (green) covering brain capillaries visualized by lectin staining (red). Scale bars $=50 \mu \mathrm{m}$. The bar graph shows pericyte coverage of brain capillaries in the cerebral cortex $(n=$ 4 mice per group; 2-4 images per animal were analyzed). The data represent the mean $\pm \mathrm{SD}$. ${ }^{*} P<0.05,{ }^{* *} P<0.01$, and ${ }^{* * *} P<0.001$ with Bonferroni's post hoc test (A, B, and $\mathbf{D})$. ${ }^{*} P<0.05$ with 2-tailed unpaired $t$ test (C).

ed by both candesartan and amlodipine (Figure $8 \mathrm{~F}$ ). No pathways closely related to matrisome protein regulation or TGF- $\beta$ signaling were found to be enriched for proteins altered by genotype or drug treatment in the GO enrichment or Kyoto Encyclopedia of Genes and Genomes (KEGG) pathway analyses (Supplemental Figure 14, Supplemental Figure 15, A-E, and Supplemental Table 16).

\section{Discussion}

Here, we showed that the cerebral arteries of HTRA1 ${ }^{-/-}$mice exhibited CARASIL-like pathological features. Although $\mathrm{HTRA}^{-/-}$mice did not show degeneration of SMCs, they exhibited intimal thickening, alteration of the elastic lamina, accumulation of matrisome proteins in the intima, and vascular enlargement. In addition, HTRA1 $^{-/-}$mice showed decreased vascular distensibility and cerebral blood flow. Most importantly, we showed that the candesartan administration substantially improved these changes.

We first discuss which changes primarily contribute to arterial dysfunction. Thickening of the intima and degeneration of the tunica media are pathological hallmarks of CARASIL $(7,9,48)$. In $\mathrm{HTRA}^{-/-}$mice, intimal thickening developed, and matrisome 
A

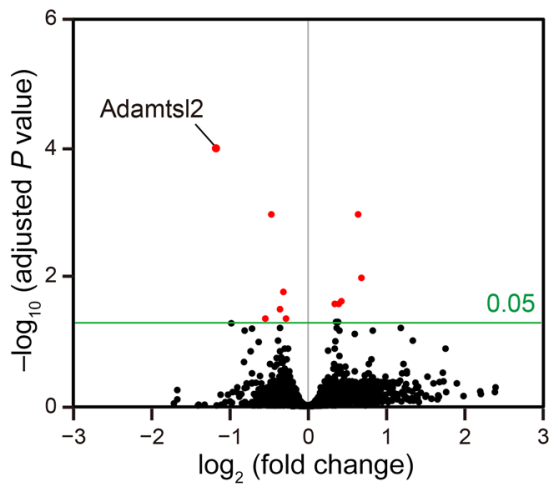

c

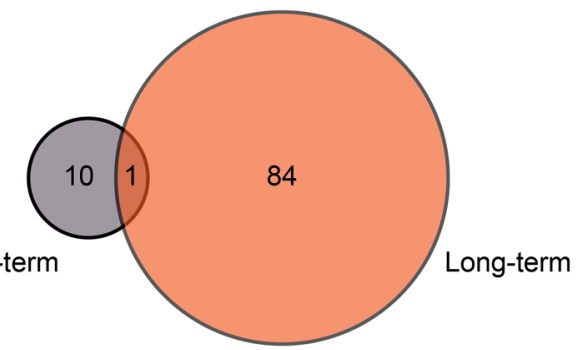

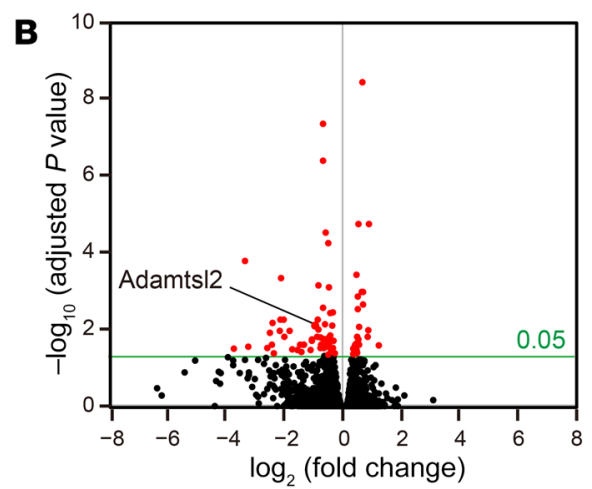

D

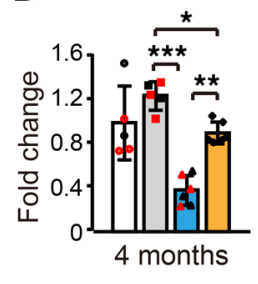

E

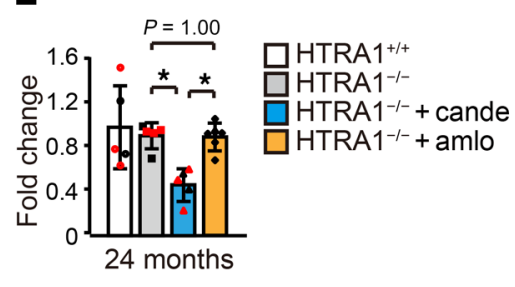

$\mathbf{F}$
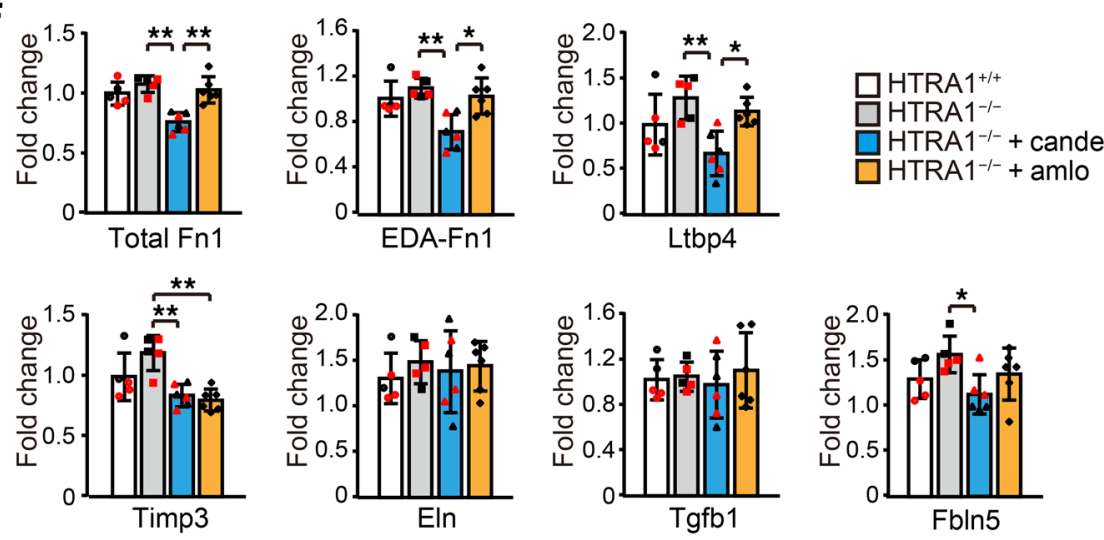

Figure 8. Candesartan treatment downregulates vascular matrisome gene expression. (A and $\mathbf{B})$ Volcano plot of gene expression in the anterior and middle cerebral arteries of untreated HTRA1 $1^{-1-}$ mice and HTRA1 ${ }^{-1-}$ mice subjected to short-term (1 week) (A) or long-term (from 4 to 24 months of age) (B) candesartan treatment according to RNA-seq analysis ( $n=3$ animals per group). The threshold for differential expression was set to an adjusted $P<$ 0.05. Data exceeding the threshold are shown in the red plot. (C) The Venn diagram illustrates a gene (Adamts/2) that overlapped between the gene sets that were differentially expressed in the cerebral arteries of $\mathrm{HTRA}^{-/-}$mice subjected to short-term and long-term candesartan treatment according to the RNA-seq analysis. ( $\mathbf{D}$ and $\mathbf{E}$ ) ddPCR analysis using an increasing number of samples confirmed the reducing effect of candesartan on Adamts/2 expression ( $n=5-6$ animals per group). A similar analysis was performed on amlodipine-treated HTRA1 ${ }^{-/-}$mouse samples. (F) We quantified the level of each mRNA by ddPCR using total RNA extracted from the arteries of 4-month-old $\mathrm{HTRA}^{+/+}$and $\mathrm{HTRA} 1^{-/-}$mice with or without 1 week candesartan or amlodipine treatment ( $n=5-6$ animals per group). The bar graphs show values relative to those for HTRA1 ${ }^{+/+}$mice. The red dots represent samples used for RNA-seq analysis, and the black dots indicated additional samples that were collected, and cDNA was prepared at the same time. The data represent the mean \pm SD. ${ }^{*} P<0.05,{ }^{* *} P<0.01$, and ${ }^{* *} P<0.001$ with Bonferroni's post hoc test $(\mathbf{D}-\mathbf{F})$.

proteins accumulated in the intima. While no degeneration of the tunica media was observed, expression of contractile genes in SMCs and pericyte coverage were decreased. Importantly, candesartan treatment alleviated the accumulation of matrisome proteins in the intima and improved vascular distensibility and cerebral blood flow. On the other hand, the decreased expression of contractile genes in SMCs and reduced pericyte coverage were not improved. In addition, the functional impact of the reduced mRNA levels of contractile genes in SMCs may be weak because these changes were not reflected in the protein levels (Supplemental Table 1 and refs. 49, 50). These results indicate that the matrisome protein accumulation contributes to arterial dysfunction in HTRA1 ${ }^{-/-}$ mice. In other organs, the accumulation of matrisome proteins was shown to increase arterial stiffness and reduce vascular distensibility, resulting in reduced blood flow (51-53). Therefore, even in HTRA1 $^{-/-}$mice, we speculate that the accumulated matrisome proteins promote stiffening of the cerebral arteries and reduce vascular distensibility, which may lead to a decrease in cerebral blood flow.

We considered which of the accumulated matrisome proteins are important in the development of arteriopathy. Decreased 
activity of HTRA1 resulted in the accumulation of its substrates FN, LTBP-4, TIMP3, fibulin-5, and TGF- $\beta 1$. Topological analysis of protein-protein interactions showed that $\mathrm{FN}$ is an important hub protein of the network of matrisome proteins accumulated in the arteries of HTRA $1^{-/-}$mice. LTBP4 binds to the TGF- $\beta$-LAP complex and is tethered to the ECM via FN $(54,55)$. TIMP3 is also a matrisome-associated protein that binds to the core matrisome, including FN $(56,57)$. In addition, LTBP-4, fibulin-5, and FN promote elastin deposition and elastic fiber assembly (58-60). Indeed, we showed that the ectopic elastic lamina was adjacent to LTBP-4 and fibulin-5 in both HTRA1 ${ }^{-/-}$mice and patients with CARASIL. The increase in elastin, which was not a substrate of HTRA1, indicates that nonsubstrate proteins also accumulate via protein-protein interactions. Thus, we speculate that the accumulation of FN, a scaffolding protein for ECM, may facilitate the accumulation of matrisome proteins $(61,62)$.

Regarding the mechanism of the inhibitory effect of candesartan on arteriopathy, candesartan but not amlodipine slightly decreased Fn1, Ltbp-4, and Fbln 5 mRNA expression. However, RNA-seq data and GO enrichment analysis did not support changes in the expression of matrisome-related genes. On the other hand, only Adamtsl 2 mRNA expression was significantly altered by candesartan in both 4-month-old and 24-month-old mice. ADAMTSL2 has been implicated in FN network formation (47), and Adamts12 knockout mice have been reported to exhibit decreased fibrillar FN content (46). Thus, we propose that by reducing Adamtsl 2 mRNA, candesartan further reduces the accumulation of $\mathrm{FN}$.

We also considered whether TGF- $\beta$ signaling contributes to the accumulation of matrisome proteins. Increased TGF- $\beta$ signaling has been identified as a cause of increased $F n 1$ mRNA expression and intimal thickening (63). Indeed, although $\mathrm{HTRA}^{-/-}$mice showed accumulation of TGF- $\beta$ s, Fn1 mRNA expression was not altered in the mice. In addition, we found no evidence of altered TGF- $\beta$ signaling in HTRA1 ${ }^{-/-}$mice by analysis of TGF- $\beta$-regulated gene expression or immunochemical and immunohistochemical analysis of canonical and noncanonical pathways. Therefore, the involvement of abnormal TGF- $\beta$ signaling is presumed to be low.

In $\mathrm{HTRA}^{-/-}$mice, matrisome protein accumulation was found in cerebral large vessels, pial arteries, and arterioles, but not in capillaries, which resembles the distribution of intimal thickening in patients with CARASIL $(9,10)$. In contrast, in patients with cerebral autosomal dominant arteriopathy with subcortical infarcts and leukoencephalopathy (CADASIL) and model mice, the Notch3 extracellular domain preferentially accumulates in capillaries (33, 64-66). This difference may be related to the fact that capillary barrier function is impaired in the CADASIL model but preserved in the CARASIL model (66-68). We propose that one of the reasons for the differences in the affected vessels is due to differences in the expression of the causative gene in each vessel. HTRA1 was expressed in endothelial cells more prominently of pial arteries than of arterioles or capillaries, which is consistent with the study of single-cell RNA-seq analysis (69) (https://endotheliomics. shinyapps.io/ec_atlas/). In contrast, Notch3 is highly expressed in pericytes on capillaries $(70,71)$. In addition, the components of matrisome proteins in the ECM differ among each type of vessel $(62,72-76)$. These differences may also be related to the vessel selectivity of matrisome protein accumulation in $\mathrm{HTRA}^{-/-}$mice.
This study has several limitations. First, we did not completely exclude the effect of accumulated proteins other than matrisome proteins. Second, the involvement of alterations in TGF- $\beta$ signaling, which is locally and transiently activated, cannot be completely ruled out (77). Moreover, it is also possible that mechanisms compensating for chronic activation may make it difficult to detect signal changes. Third, the mechanism of action of candesartan has not been fully elucidated. RNA-seq analysis of blood vessels was not able to clarify the mechanism. However, single-cell RNA-seq analysis of each component cell may provide an insight into the action of candesartan. Fourth, MS and biochemical analyses of brain parenchymal arteries were not performed. Fifth, we were not able to identify the functional consequence and the molecular mechanisms of pericyte abnormalities and conversion of SMCs to noncontractile forms. For instance, while there was reduced pericyte coverage, there were no abnormalities in blood-brain barrier function in HTRA1-/mice (44). Although we attempted to detect tracer extravasation using total brain lysates, spatially resolved analysis on brain slices might be better able to detect partial blood-brain barrier breakdown. Further study is necessary to elucidate the causes and outcomes of alterations of the mural cells. Another limitation is that HTRA1 ${ }^{-/}$mice did not recapitulate the full spectrum of symptoms exhibited by patients with CARASIL, such as white matter lesions, stroke, and loss of SMCs (7-10), which we assume to be related to the lifespan of the mice or the anatomical difference of cerebral vasculature between species. In addition, we were not able to detect a significant increase in LTBP-1 by immunoblotting analysis, whereas a 10 -fold increase was detected by MS analysis. This discrepancy may be related to technical limitations such as the solubilization efficiency of the protein from the matrisome protein accumulation area in $\mathrm{HTRA}^{-/-}$mice. Finally, in this study, MS analysis was performed on mouse samples but not on human samples. Proteomic profiling of patient samples may be useful for future studies.

This study demonstrated the importance of matrisome protein accumulation and thickening of the intima of cerebral arteries, which caused reduced vascular dispensability and cerebral blood flow in HTRA1 ${ }^{-/-}$mice. The most important finding is that matrisome protein accumulation and arteriopathy were ameliorated by candesartan. Future studies should clarify the effects of other AT1RBs and whether candesartan is effective even after arteriopathy has progressed. In addition, since the vascular accumulation of FN is also known to occur in sporadic CSVD (6), drugs that suppress FN, inhibit Adamtsl2 expression, or increase HTRA1 activity may be novel therapeutic candidates for CARASIL as well as sporadic CSVD. These approaches may open new avenues for preventing the progression of CSVD.

\section{Methods}

See Supplemental Data for additional methods.

Animals and study design. HTRA1 ${ }^{-/-}$mice were obtained from Lexicon Pharmaceuticals. HTRA1 ${ }^{-/-}$mice were backcrossed with C57BL/6JJcl mice more than 10 times. Mouse genotypes were determined by PCR using tail DNA (Supplemental Figure 1, A and B and Supplemental Table 17). HTRA1 ${ }^{+/+}$littermates were used as controls. For evaluation of cerebral blood flow, male HTRA1 ${ }^{+/+}$and $\mathrm{HTRA1}^{-/-}$ 
mice were used. In the other experiments, both female and male $\mathrm{HTRA}^{+/+}$and HTRA1 ${ }^{-/-}$mice were used to ensure an equal sex ratio.

Sample preparation for data-independent acquisition (DIA) MS analysis. For this analysis, we used 3 animals per group. Independent sample sets from different mice were used in the first and second MS analyses. Anterior and middle cerebral artery samples were precipitated in acetonitrile (ACN) containing $0.1 \%$ trifluoroacetic acid (TFA) by using a water bath-type sonicator and then centrifuged at $15,000 \mathrm{~g}$ for 15 minutes at $4^{\circ} \mathrm{C}$ to remove the supernatant. The precipitate was extracted in $0.5 \%$ sodium dodecanoate and $100 \mathrm{mM}$ Tris- $\mathrm{HCl}(\mathrm{pH}$ 8.5) by using a water bath-type sonicator. The concentrations of the extracted proteins were measured by using a BCA protein assay kit (Thermo Fisher Scientific) and adjusted to $1 \mathrm{mg} / \mathrm{mL}$ with $0.5 \%$ sodium dodecanoate and $100 \mathrm{mM}$ Tris- $\mathrm{HCl}(\mathrm{pH} \mathrm{8.5)}$. A total of $20 \mu \mathrm{g}$ protein extract was treated with $10 \mathrm{mM}$ dithiothreitol at $50^{\circ} \mathrm{C}$ for 30 minutes and then subjected to alkylation with $30 \mathrm{mM}$ iodoacetamide in the dark at room temperature for 30 minutes. The reaction with iodoacetamide was stopped with $60 \mathrm{mM}$ cysteine for 10 minutes. The mixture was diluted with $150 \mu \mathrm{L}$ of $50 \mathrm{mM}$ ammonium bicarbonate and digested by adding $1 \mu \mathrm{g}$ trypsin/Lys-C mix (Promega) overnight at $37^{\circ} \mathrm{C}$. The digested sample was acidified with $30 \mu \mathrm{L}$ of $5 \%$ TFA and then sonicated. The mixture was shaken for 5 minutes and centrifuged at 15,000 $g$ for 5 minutes. The supernatant was desalted by using C18-StageTips and then dried with a centrifugal evaporator. The dried peptides were redissolved in $3 \%$ ACN and $0.1 \%$ formic acid (FA). The redissolved peptides were measured by using a colorimetric peptide assay kit (Thermo Fisher Scientific) and transferred to a hydrophilic-coated low-adsorption vial (ProteoSave vial; AMR Inc.).

DIA-MS analysis. Pretreatment for shotgun proteome analysis was performed as previously reported (78). Peptides were directly injected onto a $75 \mu \mathrm{m} \times 12 \mathrm{~cm}$ nanoLC nanocapillary column (Nikkyo Technos Co.) at $40^{\circ} \mathrm{C}$ and then separated over an 80 -min gradient at a flow rate of $100 \mathrm{~nL} / \mathrm{min}$ using an UltiMate 3000 RSLCnano LC system (Thermo Fisher Scientific). Peptides eluted from the column were analyzed on a Q Exactive HF-X mass spectrometer (Thermo Fisher Scientific) for overlapping window DIA-MS $(78,79)$. MS1 spectra were collected in the range of 495 to $785 \mathrm{~m} / \mathrm{z}$ at 30,000 resolution, the automatic gain control target was set to $3 \times 10^{6}$, and the maximum injection time was set to 55 . MS2 spectra were collected in the range of more than $200 \mathrm{~m} / \mathrm{z}$ at 15,000 resolution, the automatic gain control target was set to $3 \times$ $10^{6}$, the maximum injection time was set to auto, and the stepped normalized collision energies were 22,26 , and 30 . The isolation width for MS2 was set to $4 \mathrm{~m} / \mathrm{z}$, and overlapping window patterns in 500 to 7780 $\mathrm{m} / \mathrm{z}$ were used as window placements optimized by Skyline (80). Raw data files of the LC-MS/MS analyses have been deposited in the ProteomeXchange Consortium (http://proteomecentral.proteomexchange. org) via the jPOST partner repository (http://jpostdb.org) with the following identifiers: jPOST Repository Database IDs, JPST000994 and JPST001052; Proteome Xchange IDs, PXD022298 and PXD023477.

MS files were searched against a mouse spectral library using Scaffold DIA (Proteome Software, Inc.). The mouse spectral library was generated from the mouse protein sequence database (UniProt id UP000000589, reviewed, canonical, 17,042 entries) by Prosit (81). The Scaffold DIA search parameters were as follows: experimental data search enzyme, trypsin; maximum missed cleavage sites, 1 ; precursor mass tolerance, $10 \mathrm{ppm}$; fragment mass tolerance, $10 \mathrm{ppm}$; and static modification, cysteine carbamidomethylation. The protein iden- tification threshold for both peptides and proteins was set to FDR of less than $1 \%$. Peptide quantification was performed with the EncyclopeDIA algorithm (82) in Scaffold DIA. For each peptide, the 4 highest quality fragment ions were selected for quantitation. Protein quantification was estimated from the summed peptide quantification. Proteins identified by 2 or more unique peptides were compared between groups. Matrisome proteins were extracted according to MatrisomeDB (http://matrisomedb.pepchem.org/) (83). GO enrichment analysis was performed with PANTHER (http://geneontology.org/). An adjusted $P$ less than 0.05 and a fold change in protein abundance greater than 1.5 or less than $1.5^{-1}$ were set as significance thresholds. To assess the relative protein abundance for different proteins, we normalized DIA protein intensity to molecular weight to correct for the higher probability of peptide identification in larger proteins $(84,85)$.

Protein-protein interaction network analysis. The protein-protein network was constructed, visualized, and topologically analyzed with Cytoscape 3.8.0 $(86,87)$. Specifically, the $\mathrm{Cc}, \mathrm{C}_{\mathrm{B}}$, and D were calculated using the Cytoscape Network Analysis module.

Vascular and brain fresh tissue collection. Blood was removed by transcardial perfusion with HBSS. Segments of the anterior and middle cerebral arteries with medium-sized branches were isolated from excised mouse brains in ice-cold MEM under a dissecting microscope, immediately frozen on dry ice, and stored at $-80^{\circ} \mathrm{C}(88)$. The cerebral cortex was dissected, and the pia matter was removed by rolling on dry filter paper.

ECM preparation. ECM was prepared according to a previously described method (89). Briefly, anterior and middle cerebral arteries were homogenized in sodium deoxycholate (DOC) lysis buffer $(150 \mathrm{mM}$ $\mathrm{NaCl}, 1 \%$ Triton X-100, 0.5\% sodium DOC, and $50 \mathrm{mM}$ Tris- $\mathrm{HCl}[\mathrm{pH}$ 7.5]) and then cleared by centrifugation. After the soluble tissue materials were collected, the insoluble fraction (ECM fraction) was homogenized in SDS lysis buffer (62.5 mM Tris-HCl [pH 6.8], 2\% SDS, and 0.5\% NP-40). After homogenization, 2-mercaptoethanol was added to the lysates at a concentration of $5 \%$, and then the lysate was incubated for 30 minutes at room temperature. After centrifugation, SDS-solubilized ECM fractions were subjected to immunoblotting as described below.

Immunoblotting. Protein samples were separated by SDS polyacrylamide gel electrophoresis and transferred onto PVDF membranes. The required volume of each ECM fraction sample was determined according to the protein content in the sodium DOC-soluble fraction. The membranes were probed with the following antibodies: mouse anti-LTBP-4 antibody (AF2885; 1:2000; R\&D Systems), rabbit anti-LTBP-1 antibody (ab78294; 1:1000; abcam), rabbit anti-FN antibody (ab2413; 1:1000; Abcam), mouse anti-EDA ${ }^{+}$FN antibody (F6140; 1:3000; Sigma), rabbit anti-elastin antibody (PR385; 1:2000; Elastin Products), goat anti-LAP- $\beta 1$ (AF-246-NA; $1: 100$; R\&D Systems), rabbit anti-TIMP3 antibody (ab58804; 1:500; Abcam), rabbit anti-fibulin-5 (ab109428; 1:1000; Abcam), rabbit anti-TGF- $\beta 2$ antibody (ab36495; 1:1000; Abcam), mouse anti- $\beta$-actin antibody (M1773; 1:5000; Medical and Biological Laboratories Co.), rabbit anti-phospho-SMAD2 antibody (3108; 1:500; Cell Signaling Technologies), rabbit anti-phospho-SMAD3 antibody (ab52903; 1:1000; Abcam), rabbit anti-phospho-SMAD1 antibody (ab214423; 1:1000; Abcam), rabbit anti-phospho-SMAD5 antibody (ab92698; 1:5000; Abcam), rabbit anti-phospho-Erk1/2 (p44/42 MAPK) antibody (4370s; 1:1000; Cell Signaling Technologies), rabbit anti-phospho-Erk5 antibody (3371S; 1:1000; Cell Signaling Technologies), rabbit anti-phospho-p38 
antibody (4511S; 1:1000; Cell Signaling Technologies), and rabbit antiSAPK/JNK antibody (4668S; 1:1000; Cell Signaling Technologies). Membrane immunoreactivity was detected by peroxidase-conjugated anti-immunoglobulin antibodies followed by chemiluminescence. Images were captured using an ImageQuant LAS-4000 apparatus (Fujifilm). Immunoreactivity was measured by densitometric analysis and normalized to $\beta$-actin levels in DOC-soluble fractions.

Drug administration. Candesartan cilexetil (Nihon Kayaku, Co. Ltd.) and amlodipine (Wako) were administered to mice via drinking water at doses with equivalent blood pressure-lowering effects beginning at 4 months of age. Candesartan and amlodipine were dissolved and diluted to final concentrations of $37.5 \mathrm{mg} / \mathrm{L}$ and $125 \mathrm{mg} / \mathrm{L}$, respectively. We previously determined that the daily water intake of adult mice ( 6 months of age) is approximately $2.5 \mathrm{~mL}$. Therefore, the dosages of candesartan and amlodipine were approximately $3 \mathrm{mg} / \mathrm{kg} /$ day and $10 \mathrm{mg} / \mathrm{kg} /$ day, respectively. These doses are comparable to those used clinically in humans to treat blood pressure (90). Both doses were titrated to achieve an approximately $15 \%$ to $20 \%$ decrease in blood pressure.

Pressure myography. Animals were anesthetized with isoflurane and decapitated, and their brains were quickly removed and placed in cold calcium-free HEPES-buffered physiological salt solution (PSS; $141.9 \mathrm{mM} \mathrm{NaCl}, 4.7 \mathrm{mM} \mathrm{KCl}, 1.2 \mathrm{mM} \mathrm{KH}_{2} \mathrm{PO}_{4}, 1.7 \mathrm{mM} \mathrm{MgSO}_{4}, 10 \mathrm{mM}$ HEPES, $0.5 \mathrm{mM}$ EDTA, $2 \mathrm{mM}$ EGTA, and $5 \mathrm{mM}$ glucose [pH 7.3]). The first branch-free segment of the middle cerebral artery was carefully isolated and mounted on glass cannulas in a pressure myograph chamber (Danish Myo Technology [DMT]). Vessels were bathed with warm oxygenated PSS at an intraluminal pressure of $80 \mathrm{mmHg}$, and the vessels were allowed to equilibrate for 30 minutes. The passive structure of vessels was assessed with increasing intraluminal pressure from 5 to $140 \mathrm{mmHg}$ in 20-mmHg increments. Vessel lumen inner diameters (IDs) were tracked and recorded after 5 minutes at each pressure step by video microscopy using Myoview software (DMT). Passive distensibility was calculated as the percent change in ID from the original diameter (ID0, at $5 \mathrm{mmHg}$ ) at a given pressure (IDp): [(IDp - IDO)] / ID0] $\times 100(\%)(91-93)$.

MRI measurements. MRI experiments were performed with an 18-cm bore $7 \mathrm{~T}$ horizontal magnet (Magnex Scientific) and a Varian Unity-INOVA-300 system (Varian Inc.) equipped with an actively shielded gradient. A birdcage transmission coil with a $6-\mathrm{cm}$ internal diameter and quadrature receiving coils were used in the study. Cerebral perfusion was assessed by continuous arterial spin labeling (CASL; ref. 94) with a centric ordered variable tip angle gradient-echo (VTE-GRE). Three seconds of radiofrequency (RF) at $\pm 10 \mathrm{~mm}$ from the imaging slice were irradiated with a $1 \mathrm{~g} / \mathrm{cm}$ axial gradient alternatively in 0.8 seconds before the VTE-GRE sequence. Sixty-four pairs of images were summed to enhance the signal-to-noise ratio. The magnetization transfer ratio (MTR) was evaluated under the same conditions used for cerebral perfusion measurements but without an axial gradient for adiabatic inversion. T1 measurements were performed using a centric ordered snapshot-FLASH with a hyperbolic secant inversion pulse (95) and a 32-point (20-5000 ms) inversion delay. Quantitative cerebral blood flow maps were obtained from the cerebral perfusion image, the T1 map, and the MTR map according to the theory described by Ewing et al. (96). The maps were generated using MRI image calculation software (MR Vision, MR Vision Co.). The animals were anesthetized with $3 \%$ to $5 \%$ isoflurane for induction followed by $1.2 \%$ isoflurane for maintenance. Cerebral blood flow in normocapnia was measured under $1 \mathrm{~L} / \mathrm{min}$ of $30 \% \mathrm{O}_{2} / 70 \% \mathrm{~N}_{2} \mathrm{O}$ under spontaneous breathing through a face mask. Hypercapnia was induced with $1 \mathrm{~L} / \mathrm{min}$ of $30 \% \mathrm{O}_{2} / 60 \% \mathrm{~N}_{2} \mathrm{O} / 10 \% \mathrm{CO}_{2}$. Cerebral blood flow in hypercapnia was measured after 10 minutes of prolonged $10 \%$ $\mathrm{CO}_{2}$ exposure following induction of hypercapnia under spontaneous breathing. The rectal temperature was maintained at $37^{\circ} \mathrm{C} \pm 0.5^{\circ} \mathrm{C}$ throughout the measurements using a homemade air conditioning system. The cerebral blood flow was assessed in the cerebral cortex and striatum. Data for the mouse white matter, which is small in size, could not be obtained by CASL due to the partial volume effect (41).

RNA-seq and analysis. For this analysis, we used 3 animals per group. Polyadenylated mRNA was enriched by an oligo-dT probe. The RNA-seq library was prepared using an Illumina TruSeq Stranded mRNA Sample Prep Kit according to the manufacturer's instructions. Quality control and quantification of the library were performed using an Agilent Bioanalyzer and a KAPA Library Quantification Kit (KAPA Biosystems). All 9 libraries were pooled and sequenced on the Illumina HiSeq 2500 platform (high output mode, 50-base single read) on a single flow cell lane. Raw reads were adapter trimmed and quality filtered using the FASTX-Toolkit (http://hannonlab.cshl. edu/fastx_toolkit/index.html), and resulting reads with $\mathrm{N}$ and those derived from ribosomal RNA were excluded. The clean reads were mapped to the mouse reference genome sequence (GRCm38/mm10) using TopHat (97). Mapped reads were counted with HTseq (98). We used DEseq2 to identify differentially expressed genes with an adjusted $P$ less than 0.05 (99). Genes containing samples with a read count of 0 were excluded from subsequent analyses. GO enrichment analysis was performed with PANTHER (http://geneontology.org/). KEGG pathway enrichment analyses were performed with the clusterProfiler R package (100). These data are available from the DDBJ Sequence Read Archive (https://ddbj.nig.ac.jp/DRASearch/) (accession numbers DRA011075 and DRA011076).

Statistics. Statistical computation was performed using IBM SPSS 22. The data were first subjected to the Shapiro-Wilk test (for fitting to the Gaussian distribution) and Levene's test (for equal variance). Oneway ANOVA, 2-way repeated ANOVA, or 2-tailed unpaired $t$ test was used for data with a Gaussian distribution and equal variance. Subsequently, the data were analyzed by the Bonferroni's post hoc test. Alternatively, data with unequal variance were analyzed by the SteelDwass test or Mann-Whitney $U$ test. $P$ less than 0.05 was regarded as statistically significant. The number of mice used for each analysis is indicated in the figure legends. All data are presented as the mean \pm SD. DEseq2 was used to identify differentially expressed genes in the RNA-seq data with an adjusted $P$ less than $0.05(99,101)$. Differential protein levels from DIA-MS proteomics were analyzed using ROPECA with FDR cutoff set to $0.05(102,103)$.

Study approval. All animal experiments described were approved by the Animal Use and Care Committee of Niigata University and followed the guidelines of the National Institutes of Health. The human study was approved by the Institutional Ethical Review Board of Niigata University. Written informed consent was obtained from the families, and all clinical investigations were conducted according to the principles expressed in the Declaration of Helsinki.

\section{Author contributions}

TK designed the research study, conducted the experiments, acquired the data, analyzed the data and wrote the manuscript. 
RM, YS, NF, SH, TS, AK, YK, and MU conducted the experiments and acquired and analyzed the data. SS, FK, and HI conducted the experiments, acquired and analyzed the data and wrote the manuscript. MF, MM, and $\mathrm{YH}$ analyzed the data and provided expertise related to the experiments. YT and AK designed the research study and provided human materials and expertise related to the experiments. AS, RS, SA, MK, and AS designed the research study and wrote the manuscript. MH, MI, and MN designed the research study and provided expertise related to experiments. HK and SM provided human materials. HN and ST designed the research study. OO designed the research study, analyzed the data and wrote the manuscript. All authors participated in interpreting the results and revising the manuscript.

\section{Acknowledgments}

This work was supported by a grant-in-aid for Scientific Research on Innovative Areas (Brain Protein Aging and Dementia Control; 26117006) from MEXT; a grant-in-aid for Practical Research Projects for Rare/Intractable Diseases (JP19ek0109236h0003) and Strategic Research Program for Brain Sciences (19dm0107106) from AMED; a grant-in-aid for Scientific Research (A) (16H02656), a grant-in-aid for Scientific
Research (C) (17K09749), a grant-in-aid for Young Scientists (B) (26860208), a grant-in-aid for Scientific Research on Innovative Areas-Resource and technical support platforms for promoting research "Advanced Bioimaging Support" (JP16H06280), and a grant-in-aid for Scientific Research on Innovative AreasPlatforms for Advanced Technologies and Research Resources (16H06277) from the Japan Society for the Promotion of Science; a grant-in-aid for Medical Research from the Takeda Science Foundation; and a grant-in-aid for Research on Intractable Disease from the Japanese Ministry of Health, Labor, and Welfare, Japan. This work was supported by the Ministry of Education, Culture, Sports, Science, and Technology in Japan (research grant to RIKEN Center for Integrative Medical Sciences, Laboratory for Comprehensive Genomic Analysis). We thank Rei Kawashima for RNA-seq library preparation; the Sequence Platform of RIKEN IMS for sequencing; and Chitose Takahashi, Michihira Tagami, and Yasushi Okazaki for helpful discussion about RNA-seq data analysis.

Address correspondence to: Osamu Onodera, 1-757 Asahimachi-dori, Chuo-Ku, Niigata City, Niigata 951-8585, Japan. Phone: 81.25.227.0684; E-mail: onodera@bri.niigata-u.ac.jp.
1. Pantoni L. Cerebral small vessel disease: from pathogenesis and clinical characteristics to therapeutic challenges. Lancet Neurol. 2010;9(7):689-701.

2. Wardlaw JM, et al. Mechanisms of sporadic cerebral small vessel disease: insights from neuroimaging. Lancet Neurol. 2013;12(5):483-497.

3. Hara K, et al. Association of HTRA1 mutations and familial ischemic cerebral small-vessel disease. N Engl J Med. 2009;360(17):1729-1739.

4. Nozaki H, et al. Distinct molecular mechanisms of HTRA1 mutants in manifesting heterozygotes with CARASIL. Neurology. 2016;86(21):1964-1974.

5. Fukutake T. Cerebral autosomal recessive arteriopathy with subcortical infarcts and leukoencephalopathy (CARASIL): from discovery to gene identification. J Stroke Cerebrovasc Dis. 2011;20(2):85-93.

6. Okeda R, et al. Pathology of the cerebral artery in Binswanger's disease in the aged: observation by serial sections and morphometry of the cerebral arteries. Neuropathology. 2004;24(1):21-29.

7. Oide T, et al. Extensive loss of arterial medial smooth muscle cells and mural extracellular matrix in cerebral autosomal recessive arteriopathy with subcortical infarcts and leukoencephalopathy (CARASIL). Neuropathology. 2008;28(2):132-142.

8. Yanagawa S, et al. Cerebral autosomal recessive arteriopathy with subcortical infarcts and leukoencephalopathy. Neurology. 2002;58(5):817-820.

9. Ito $\mathrm{S}$, et al. Histopathologic analysis of cerebral autosomal recessive arteriopathy with subcortical infarcts and leukoencephalopathy (CARASIL): a report of a new genetically confirmed case and comparison to 2 previous cases. J Neuropathol Exp Neurol. 2016;75(11):1020-1030.

10. Ito J, et al. Histopathologic features of an autopsied patient with cerebral small vessel disease and a heterozygous HTRA1 mutation [published online May 25, 2018]. Neuropathology. https:// doi.org/10.1111/neup.12473.

11. Joutel A, et al. Perturbations of the cerebrovascular matrisome: A convergent mechanism in small vessel disease of the brain? JCereb Blood Flow Metab. 2016;36(1):143-157.

12. Naba A, et al. The extracellular matrix: tools and insights for the "omics" era. Matrix Biol. 2016;49:10-24.

13. Zellner A, et al. CADASIL brain vessels show a HTRA1 loss-of-function profile. Acta Neuropathol. 2018;136(1):111-125.

14. Naba A, et al. The matrisome: in silico definition and in vivo characterization by proteomics of normal and tumor extracellular matrices. $\mathrm{Mol}$ Cell Proteomics. 2012;11(4):M111.014647.

15. Naba A, et al. Towards definition of an ECM parts list: an advance on GO categories. Matrix Biol. 2012;31(7-8):371-372.

16. Shiga A, et al. Cerebral small-vessel disease protein HTRA1 controls the amount of TGF- $\beta 1$ via cleavage of proTGF- $\beta 1$. Hum Mol Genet. 2011;20(9):1800-1810.

17. Grau S, et al. The role of human HtrA1 in arthritic disease. J Biol Chem. 2006;281(10):6124-6129.

18. Oka C, et al. HtrA1 serine protease inhibits signaling mediated by Tgfbeta family proteins. Development. 2004;131(5):1041-1053.

19. Launay S, et al. HtrA1-dependent proteolysis of TGF-beta controls both neuronal maturation and developmental survival. Cell Death Differ. 2008;15(9):1408-1416.

20. Beaufort N, et al. Cerebral small vessel disease-related protease HtrA1 processes latent TGF- $\beta$ binding protein 1 and facilitates TGF- $\beta$ signaling. Proc Natl Acad Sci U S A. 2014;111(46):16496-16501.

21. Low EL, et al. TGF $\beta$, smooth muscle cells and coronary artery disease: a review. Cell Signal. 2019;53:90-101.
22. Graham JR, et al. Serine protease HTRA1 antagonizes transforming growth factor- $\beta$ signaling by cleaving its receptors and loss of HTRA1 in vivo enhances bone formation. PLoS One. 2013;8(9):e74094.

23. Zhang L, et al. High temperature requirement factor A1 (HTRA1) gene regulates angiogenesis through transforming growth factor- $\beta$ family member growth differentiation factor 6. J Biol Chem. 2012;287(2):1520-1526.

24. Chen J, et al. BMP-responsive protease HtrA1 Is differentially expressed in astrocytes and regulates astrocytic development and injury response. J Neurosci. 2018;38(15):3840-3857.

25. Ikawati M, et al. Loss of HtrA1 serine protease induces synthetic modulation of aortic vascular smooth muscle cells. PLoS One. 2018;13(5):e0196628.

26. Arumugam S, et al. Angiotensin receptor blockers: Focus on cardiac and renal injury. Trends Cardiovasc Med. 2016;26(3):221-228.

27. Sekine $\mathrm{S}$, et al. Possible involvement of mitogen-activated protein kinase in the angiotensin II-induced fibronectin synthesis in renal interstitial fibroblasts. Arch Biochem Biophys. 2003;415(1):63-68.

28. Lanz TV, et al. Angiotensin II sustains brain inflammation in mice via TGF-beta. J Clin Invest. 2010;120(8):2782-2794.

29. Bar-Klein G, et al. Losartan prevents acquired epilepsy via TGF- $\beta$ signaling suppression. Ann Neurol. 2014;75(6):864-875.

30. Ongali B, et al. Transforming growth factor- $\beta 1$ induces cerebrovascular dysfunction and astrogliosis through angiotensin II type 1 receptormediated signaling pathways. Can J Physiol Pharmacol. 2018;96(5):527-534.

31. Barabási AL, et al. Network medicine: a network-based approach to human disease. Nat Rev Genet. 2011;12(1):56-68. 
32. Peng Q, Schork NJ. Utility of network integrity methods in therapeutic target identification. Front Genet. 2014;5:12.

33. Monet-Lepretre M, et al. Abnormal recruitment of extracellular matrix proteins by excess Notch 3 ECD: a new pathomechanism in CADASIL. Brain. 2013;136(pt 6):1830-1845.

34. Vierkotten S, et al. Overexpression of HTRA1 leads to ultrastructural changes in the elastic layer of Bruch's membrane via cleavage of extracellular matrix components. PLoS One. 2011;6(8):e22959.

35. Himeno $\mathrm{H}$, et al. Angiotensin II alters aortic fibronectin independently of hypertension. Hypertension. 1994;23(6 pt 2):823-826.

36. Verdecchia P, et al. Beyond blood pressure: evidence for cardiovascular, cerebrovascular, and renal protective effects of renin-angiotensin system blockers. Ther Adv Cardiovasc Dis. 2012;6(2):81-91.

37. Volpe M, et al. Angiotensin-II receptor blockers: benefits beyond blood pressure reduction? J Hum Hypertens. 2005;19(5):331-339.

38. Brown $\mathrm{L}$, et al. Reversal of cardiac fibrosis in deoxycorticosterone acetate-salt hypertensive rats by inhibition of the renin-angiotensin system. JAm Soc Nephrol. 1999;10(suppl 11):S143-S148.

39. Obata J, et al. Candesartan prevents the progression of glomerulosclerosis in genetic hypertensive rats. Kidney Int Suppl. 1997;63:S229-S231.

40. Habashi JP, et al. Angiotensin II type 2 receptor signaling attenuates aortic aneurysm in mice through ERK antagonism. Science. 2011;332(6027):361-365.

41. González Ballester MA, et al. Estimation of the partial volume effect in MRI. Med Image Anal. 2002;6(4):389-405.

42. Kono Y, et al. Heterozygous HTRA1 mutations with mimicking symptoms of CARASIL in two families. Clin Neurol Neurosurg. 2018;172:174-176.

43. Nozaki H, et al. Features of cerebral autosomal recessive arteriopathy with subcortical infarcts and leukoencephalopathy. Stroke. 2014;45(11):3447-3453.

44. Armulik A, et al. Pericytes regulate the bloodbrain barrier. Nature. 2010;468(7323):557-561

45. Fang Z, Cui X. Design and validation issues in RNA-seq experiments. Brief Bioinform. 2011;12(3):280-287.

46. Delhon L, et al. Impairment of chondrogenesis and microfibrillar network in Adamtsl2 deficiency. FASEB J. 2019;33(2):2707-2718.

47. Hubmacher D, Apte SS. ADAMTS proteins as modulators of microfibril formation and function. Matrix Biol. 2015;47:34-43.

48. Arima K, et al. Cerebral arterial pathology of CADASIL and CARASIL (Maeda syndrome). Neuropathology. 2003;23(4):327-334.

49. Dobnikar L, et al. Disease-relevant transcriptional signatures identified in individual smooth muscle cells from healthy mouse vessels. Nat Commun. 2018;9(1):4567.

50. Liu Y, et al. On the dependency of cellular protein levels on mRNA abundance. Cell. 2016;165(3):535-550.

51. Leung MC, et al. Aortic stiffness affects the coronary blood flow response to percutaneous coronary intervention. Am J Physiol Heart Circ Physiol.
2006;290(2):H624-H630.

52. Nelson AJ, et al. Aortic distensibility is associated with both resting and hyperemic coronary blood flow. Am J Physiol Heart Circ Physiol. 2019;317(4):H811-H819.

53. Rizzoni D, et al. Prognostic significance of small-artery structure in hypertension. Circulation. 2003;108(18):2230-2235.

54. Kantola AK, et al. Fibronectin and heparin binding domains of latent TGF-beta binding protein (LTBP)- 4 mediate matrix targeting and cell adhesion. Exp Cell Res. 2008;314(13):2488-2500.

55. Zhou Y, et al. Latent transforming growth factorbeta-binding protein- 4 regulates transforming growth factor-beta1 bioavailability for activation by fibrogenic lung fibroblasts in response to bleomycin. Am J Pathol. 2009;174(1):21-33

56. Arpino $\mathrm{V}$, et al. The role of TIMPs in regulation of extracellular matrix proteolysis. Matrix Biol. 2015;44-46:247-254.

57. Lee $\mathrm{MH}$, et al. Identification of the extracellular matrix (ECM) binding motifs of tissue inhibitor of metalloproteinases (TIMP)-3 and effective transfer to TIMP-1. J Biol Chem. 2007;282(9):6887-6898.

58 . Noda K, et al. Latent TGF- $\beta$ binding protein 4 promotes elastic fiber assembly by interacting with fibulin-5. Proc Natl Acad Sci U S A. 2013;110(8):2852-2857.

59. Pezzoli D, et al. Fibronectin promotes elastin deposition, elasticity and mechanical strength in cellularised collagen-based scaffolds. Biomaterials. 2018;180:130-142.

60. Yanagisawa $\mathrm{H}$, et al. Fibulin-5 is an elastin-binding protein essential for elastic fibre development in vivo. Nature. 2002;415(6868):168-171.

61. Singh P, et al. Assembly of fibronectin extracellular matrix. Annu Rev Cell Dev Biol. 2010;26:397-419.

62. Mouw JK, et al. Extracellular matrix assembly: a multiscale deconstruction. Nat Rev Mol Cell Biol. 2014;15(12):771-785.

63. Ruiz-Ortega M, et al. TGF-beta signaling in vascular fibrosis. Cardiovasc Res. 2007;74(2):196-206.

64. Yamamoto $Y$, et al. Brain microvascular accumulation and distribution of the NOTCH3 ectodomain and granular osmiophilic material in CADASIL. JNeuropathol Exp Neurol. 2013;72(5):416-431.

65. Lewandowska E, et al. Capillary vessel wall in CADASIL angiopathy. Folia Neuropathol. 2010;48(2):104-115.

66. Ghosh M, et al. Pericytes are involved in the pathogenesis of cerebral autosomal dominant arteriopathy with subcortical infarcts and leukoencephalopathy. Ann Neurol. 2015;78(6):887-900.

67. Pfefferkorn T, et al. Reduced cerebrovascular $\mathrm{CO}(2)$ reactivity in CADASIL: a transcranial Doppler sonography study. Stroke. 2001;32(1):17-21.

68. Joutel A, et al. Cerebrovascular dysfunction and microcirculation rarefaction precede white matter lesions in a mouse genetic model of cerebral ischemic small vessel disease. J Clin Invest. 2010;120(2):433-445

69. Kalucka J, et al. Single-cell transcriptome atlas of murine endothelial cells. Cell. 2020;180(4):764-779.

70. Nadeem T, et al. Deficiency of Notch signaling in pericytes results in arteriovenous malformations. JCI Insight. 2020;5(21):e125940.
71. Wang Y, et al. Notch3 establishes brain vascular integrity by regulating pericyte number. Development. 2014;141(2):307-317.

72. Amatschek S, et al. Blood and lymphatic endothelial cell-specific differentiation programs are stringently controlled by the tissue environment. Blood.2007;109(11):4777-4785.

73. Calabria AR, Shusta EV. A genomic comparison of in vivo and in vitro brain microvascular endothelial cells. J Cereb Blood Flow Metab. 2008;28(1):135-148.

74. Ibrahim AM, et al. Fibulin-2 is required for basement membrane integrity of mammary epithelium. Sci Rep. 2018;8(1):14139.

75. Thomsen MS, et al. The vascular basement membrane in the healthy and pathological brain. JCereb Blood Flow Metab. 2017;37(10):3300-3317.

76. Vanlandewijck M, et al. A molecular atlas of cell types and zonation in the brain vasculature. Nature. 2018;554(7693):475-480.

77. Constam DB. Regulation of TGF $\beta$ and related signals by precursor processing. Semin Cell Dev Biol. 2014;32:85-97.

78. Kawashima Y, et al. Optimization of data-independent acquisition mass spectrometry for deep and highly sensitive proteomic analysis. Int J Mol Sci. 2019;20(23):E5932.

79. Amodei D, et al. Improving precursor selectivity in data-independent acquisition using overlapping windows. J Am Soc Mass Spectrom. 2019;30(4):669-684

80. MacLean B, et al. Skyline: an open source document editor for creating and analyzing targeted proteomics experiments. Bioinformatics. 2010;26(7):966-968.

81. Gessulat S, et al. Prosit: proteome-wide prediction of peptide tandem mass spectra by deep learning. Nat Methods. 2019;16(6):509-518.

82. Searle BC, et al. Chromatogram libraries improve peptide detection and quantification by data independent acquisition mass spectrometry. Nat Commun. 2018;9(1):5128.

83. Shao X, et al. MatrisomeDB: the ECM-protein knowledge database. Nucleic Acids Res. 2020;48(d1):D1136-D1144.

84. Sachs AN, et al. LC-MS/MS analysis of differential centrifugation fractions from native inner medullary collecting duct of rat. Am J Physiol Renal Physiol. 2008;295(6):F1799-F1806.

85. Wang Z, et al. Structure of the marine siphovirus TW1: evolution of capsid-stabilizing proteins and tail spikes. Structure. 2018;26(2):238-248.

86. Shannon P, et al. Cytoscape: a software environment for integrated models of biomolecular interaction networks. Genome Res. 2003;13(11):2498-2504.

87. Yeung N, et al. Exploring biological networks with Cytoscape software. Curr Protoc Bioinformatics. 2008; Chapter 8:Unit 8.13.

88. Hur JC, et al. Protocol for isolating the mouse circle of willis. J Vis Exp. 2016;(116):54352.

89. Tatti O, et al. MT1-MMP releases latent TGFbeta1 from endothelial cell extracellular matrix via proteolytic processing of LTBP-1. Exp Cell Res. 2008;314(13):2501-2514

90. Heran BS, et al. Blood pressure lowering efficacy of angiotensin receptor blockers for primary hypertension. Cochrane Database Syst Rev. 
2008;(4):CD003822.

91. Chan SL, et al. Effect of PPAR $\gamma$ inhibition during pregnancy on posterior cerebral artery function and structure. Front Physiol. 2010;1:130.

92. Matin N, et al. DOCA-salt hypertension impairs artery function in rat middle cerebral artery and parenchymal arterioles. Microcirculation. 2016;23(7):571-579.

93. Pires PW, et al. Doxycycline, a matrix metalloprotease inhibitor, reduces vascular remodeling and damage after cerebral ischemia in stroke-prone spontaneously hypertensive rats. Am J Physiol Heart Circ Physiol. 2011;301(1):H87-H97.

94. Williams DS, et al. Magnetic resonance imaging of perfusion using spin inversion of arterial water. Proc Natl Acad Sci U S A. 1992;89(1):212-216.
95. Gowland PA, et al. The use of an improved inversion pulse with the spin-echo/inversion-recovery sequence to give increased accuracy and reduced imaging time for T1 measurements. Magn Reson Med.1989;12(2):261-267.

96. Ewing JR, et al. Direct comparison of local cerebral blood flow rates measured by MRI arterial spin-tagging and quantitative autoradiography in a rat model of experimental cerebral ischemia. JCereb Blood Flow Metab. 2003;23(2):198-209.

97. Trapnell C, et al. TopHat: discovering splice junctions with RNA-Seq. Bioinformatics. 2009;25(9):1105-1111.

98. Anders S, et al. HTSeq--a Python framework to work with high-throughput sequencing data. Bioinformatics. 2015;31(2):166-169.
99. Love MI, et al. Moderated estimation of fold change and dispersion for RNA-seq data with DESeq2. Genome Biol. 2014;15(12):550.

100.Yu G, et al. clusterProfiler: an R package for comparing biological themes among gene clusters. OMICS. 2012;16(5):284-287.

101.Langley SR, Mayr M. Comparative analysis of statistical methods used for detecting differential expression in label-free mass spectrometry proteomics. J Proteomics. 2015;129:83-92.

102. Dowell JA, et al. Benchmarking quantitative performance in label-free proteomics. ACS Omega. 2021;6(4):2494-2504.

103. Suomi T, Elo LL. Enhanced differential expression statistics for data-independent acquisition proteomics. Sci Rep. 2017;7(1):5869. 